\title{
NF-KB/MAPK activation underlies ACVR1-mediated inflammation in human heterotopic ossification
}

Emilie Barruet, ${ }^{1}$ Blanca M. Morales, ${ }^{1}$ Corey J. Cain, ${ }^{1}$ Amy N. Ton, ${ }^{1}$ Kelly L. Wentworth, ${ }^{1}$ Tea V. Chan, ${ }^{1}$ Tania A. Moody, ${ }^{1}$ Mariëlle C. Haks, ${ }^{2}$ Tom H.M. Ottenhoff, ${ }^{2}$ Judith Hellman, ${ }^{3}$ Mary C. Nakamura, ${ }^{4}$ and Edward C. Hsiao ${ }^{1}$

'Division of Endocrinology and Metabolism, Department of Medicine, and the Institute for Human Genetics, UCSF, San Francisco, California, USA. 'Leiden University Medical Center, Department of Infectious Diseases, Leiden, Netherlands. ${ }^{3}$ Department of Anesthesia and Perioperative Care, UCSF, San Francisco, California, USA. ${ }^{4}$ Division of Rheumatology, Department of Medicine, San Francisco VA Health Care System, UCSF, San Francisco, California, USA.

BACKGROUND. Inflammation helps regulate normal growth and tissue repair. Although bone morphogenetic proteins (BMPs) and inflammation are known contributors to abnormal bone formation, how these pathways interact in ossification remains unclear.

METHODS. We examined this potential link in patients with fibrodysplasia ossificans progressiva (FOP), a genetic condition of progressive heterotopic ossification caused by activating mutations in the Activin A type I receptor (ACVR1/ALK2). FOP patients show exquisite sensitivity to trauma, suggesting that BMP pathway activation may alter immune responses. We studied primary blood, monocyte, and macrophage samples from control and FOP subjects using multiplex cytokine, gene expression, and protein analyses; examined $\mathrm{CD}_{14} 4^{+}$primary monocyte and macrophage responses to TLR ligands; and assayed BMP, TCF- $\beta$ activated kinase 1 (TAK1), and NF-KB pathways.

RESULTS. FOP subjects at baseline without clinically evident heterotopic ossification showed increased serum IL-3, IL-7, IL-8, and IL-10. CD14+ primary monocytes treated with the TLR4 activator LPS showed increased CCL5, CCR7, and CXCL10; abnormal cytokine/chemokine secretion; and prolonged activation of the NF- $\mathrm{KB}$ pathway. FOP macrophages derived from primary monocytes also showed abnormal cytokine/chemokine secretion, increased TCF- $\beta$ production, and $p 38$ MAPK activation. Surprisingly, SMAD phosphorylation was not significantly changed in the FOP monocytes/macrophages.

Conflict of interest: ECH, TVC, and KLW receive clinical trial research funding support from Clementia Pharmaceuticals. ECH received clinical trial research funding support from Regeneron Pharmaceuticals and serves in an unpaid capacity on the International FOP Association Medical Registry Advisory Board, on the International Clinical Council on FOP, and on the Fibrous Dysplasia Foundation Medical Advisory Board.

License: Copyright 2018, American Society for Clinical Investigation.

Submitted: June 19, 2018

Accepted: October 11, 2018

Published: November 15, 2018

Reference information: JCI Insight. 2018;3(22):e122958. https://doi.org/10.1172/jci. insight.122958.
CONCLUSIONS. Abnormal ACVR1 activity causes a proinflammatory state via increased NF- $\kappa B$ and p38MAPK activity. Similar changes may contribute to other types of heterotopic ossification, such as in scleroderma and dermatomyositis; after trauma; or with recombinant BMP-induced bone fusion. Our findings suggest that chronic antiinflammatory treatment may be useful for heterotopic ossification.

\section{Introduction}

Inflammation is a critical regulator of normal growth and tissue repair $(1,2)$ including in normal bone homeostasis $(3,4)$ and fracture repair (5). In addition, diseases affecting the immune system can have significant effects on bone homeostasis, such as in heterotopic ossification, where inappropriate bone formation occurs at an anatomically incorrect site. Innate immunity is thought to be a key contributor to heterotopic ossification induction and formation $(6,7)$, particularly after acute injury or trauma $(7,8)$. However, the diversity of triggers and responding cell types pose challenges for elucidating how inflammatory stimuli contribute to heterotopic ossification.

Tissue trauma and the initiation of heterotopic ossification appear closely linked. Patients receiving total hip replacement or spinal surgeries are at high risk of developing heterotopic ossification, with reports as high as $47 \%$ incidence in some studies $(9,10)$. Heterotopic ossification can occur in patients with rheumatic diseases, such as scleroderma and dermatomyositis; after blast injuries; with 
severe burns; after traumatic brain injuries or strokes; or after major nonneurological surgeries, such as hip fracture repair (8-13). In some conditions, heterotopic bone can even form at anatomic sites distant from the original injury (14), suggesting that systemic factors could mediate the abnormal ossification. Furthermore, inappropriate activation of osteogenic pathways can occur in common conditions of tissue injury, such as atherosclerosis or vascular calcification, where activated bone morphogenetic protein (BMP) signaling pathways may be present (15). Studies also indicate that TGF- $\beta$ superfamily members, including BMPs and activins, are produced at high levels in multiple types of inflammation $(16,17)$ and may modulate inflammation. However, the wide variety of triggers and diversity of ligands makes it challenging to elucidate how immune function and BMP signaling converge in heterotopic ossification.

Several lines of evidence indicate key roles for inflammation in heterotopic ossification. In humans, inflammatory markers such as IL-3 and IL-12p70 have been linked to heterotopic ossification in combat wounds (18). IL-6, IL-10, monocyte chemoattractant (MCP1), effluent IP10 (IFN- $\gamma$-induced protein), and macrophage inflammatory protein $1 \alpha($ MIP1- $\alpha)$ are also associated with heterotopic ossification from high-energy trauma (19). Antiinflammatory treatments like glucocorticoids and nonsteroidal antiinflammatory drugs (NSAIDs) are the mainstay of current treatment for heterotopic ossification (20-23). Although these strategies attempt to mitigate the inflammation and subsequent bone formation, these drugs show low efficacy in blocking heterotopic ossification.

Mouse models of heterotopic ossification also show that macrophages and TGF- $\beta$ are important regulators of heterotopic ossification $(24,25)$. Trauma-induced heterotopic ossification from hind limb Achilles' tenotomy and dorsal burns in mice cause increased levels of TNF- $\alpha$ and IL-1 $\beta$ within 48 hours after injury, with persistent MCP1 and VEGF elevations detectable in saliva 1 week after injury (26). In addition, macrophages and other innate immune cells are needed for initiation and progression of fracture repair in mice (27).

Genetic conditions of heterotopic ossification can provide a more controlled venue for dissecting mechanistic contributors, allowing researchers to bypass the diversity of triggers and focus on the underlying molecular and cellular pathways. Fibrodysplasia ossificans progressiva (FOP) is a disease of massive heterotopic ossification that can occur after trauma or infection (28). FOP is caused by a highly recurring R206H (c.617>A) mutation in the Activin A type I receptor (ACVR1/ALK2), which increases signaling in response to BMPs (29). An abnormal response to Activin A mimicking a BMP-like response has also been identified $(30,31)$; however, endothelial cells derived from induced pluripotent stem cells made from FOP patients showed no increased Activin A response (32), suggesting cell-type specificity of the ACVR1 R206H neoreceptor activity to Activin A. FOP patients develop flares characterized by significant inflammation at the sites of future heterotopic ossification, with local warmth, redness, and dramatic swelling (Figure 1) (33). The bone formation in FOP is thought to recapitulate endochondral ossification and is similar to other forms of traumatic heterotopic ossification; thus, FOP may serve as a model for other diseases of human bone formation.

Macrophages are abundant in the developing heterotopic ossification lesions of chimeric FOP ACVR1 R206H mice, even in the absence of known traumatic injury (i.e., spontaneous heterotopic ossification) (34). These injury-recruited macrophages may mediate the injury response or potentially trigger heterotopic ossification, since depletion of macrophages and mast cells can reduce heterotopic ossification in FOP mouse models (24). Here, we elucidate the proinflammatory and monocyte/ macrophage changes in humans with FOP to understand how ACVR1 signaling leads to abnormal immune activation.

\section{Results}

Elevated proinflammatory and myeloid cytokines in serum of FOP patients. Obtaining blood from FOP patients is challenging. FOP is rare (prevalence of 1 in 1,300,000 to 1 in 2,000,000 worldwide; refs. 35, 36), and patients have difficulty traveling to research sites due to their immobility. Blood draws can lead to heterotopic ossification at the phlebotomy site, and often only small quantities of blood can be obtained because of difficult venous access from contractures. We were fortunate to study a cohort of FOP patients and their families seen from 2014-2018 at the UCSF Metabolic Bone Clinic using protocols approved by the UCSF Committee on Human Research (Figure 1). All FOP subjects in this study were genotyped and confirmed to carry the classical ACVR1 R206H mutation (data not shown). 


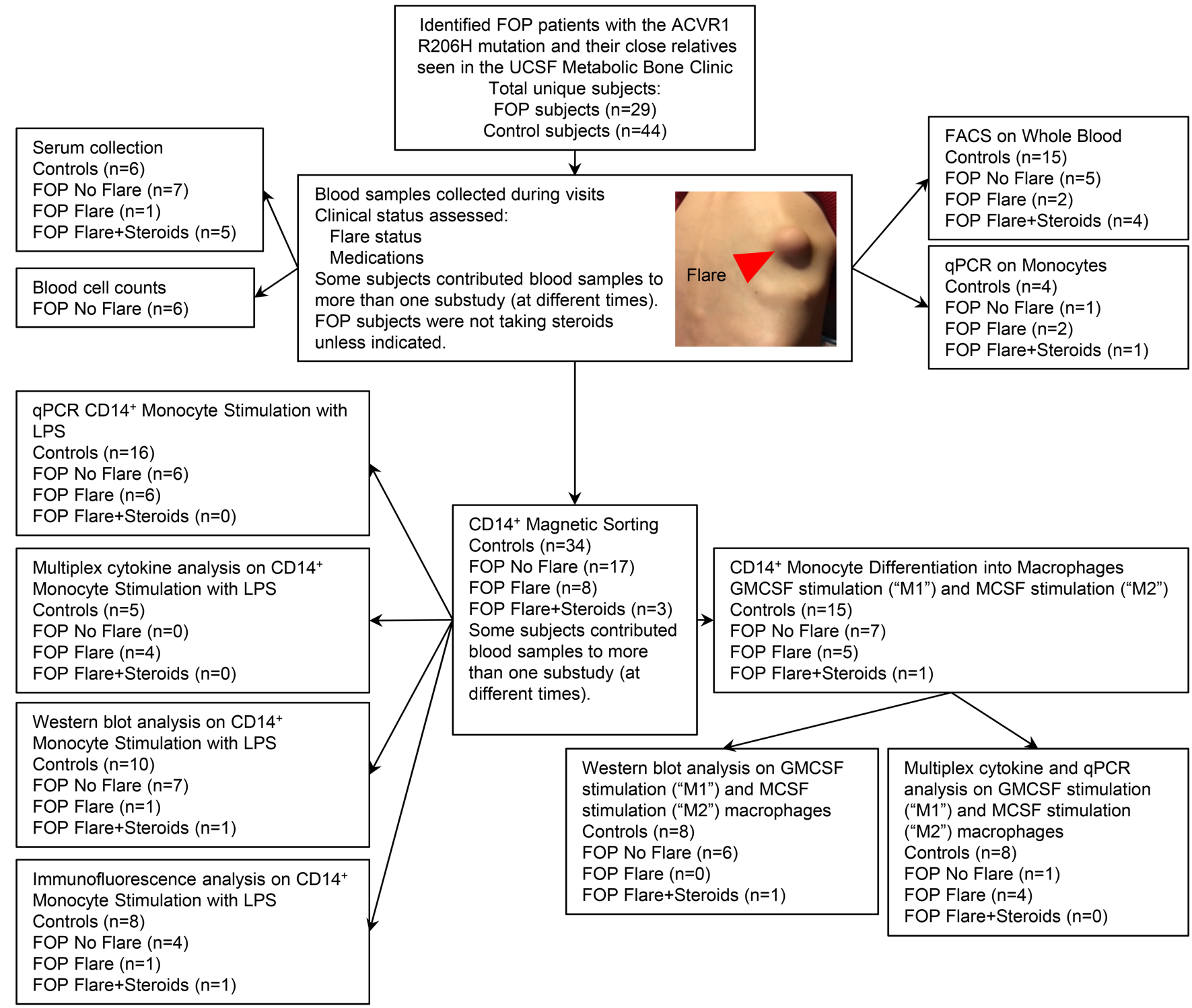

Figure 1. Patient samples flow diagram. Picture represents a flare from a FOP patient. Red arrow indicates the flare site over the right scapula. FOP subjects with no clinically evident flare were termed FOP No Flare. FOP subjects with a documented clinically ongoing flare and who were receiving standard-of-care glucocorticoid treatment for their flare at the time of the blood draw were termed FOP Flare+Steroids. Flaring FOP subjects who were not yet receiving steroids were termed FOP Flare. Control family subjects (either siblings or parents) were termed Control Subjects.

We first carefully collected serum from FOP subjects at baseline without a reported flare or clinically active bone formation (Supplemental Table 1; supplemental material available online with this article; https://doi.org/10.1172/jci.insight.122958DS1). These subjects were termed FOP No Flare. FOP subjects with a documented clinically ongoing flare (based on the presence of 2 or more symptoms, including pain, swelling, erythema, or loss of motion at a joint adjacent to the flare; Figure 1) and who were receiving standard-of-care glucocorticoid treatment (20) for their flare at the time of the blood draw were termed FOP Flare+Steroids. Control family subjects (either siblings or parents) were termed Control Subjects. Flaring FOP subjects who were not on steroids, such as FOP31, were termed FOP Flare. Additional FOP Flare subjects not receiving steroids were unavailable at the time of this initial cytokine analysis.

We performed multiplex analysis for 69 cytokines on the serum samples because of the small volumes of blood samples we received (Figure 2). Principal component analysis (PCA) showed that the FOP No Flare group showed differences from the controls but that the flaring FOP subjects narrowed the clustering back toward the controls (Figure 2A). The cytokine profiles of control, FOP No Flare, and FOP 
A

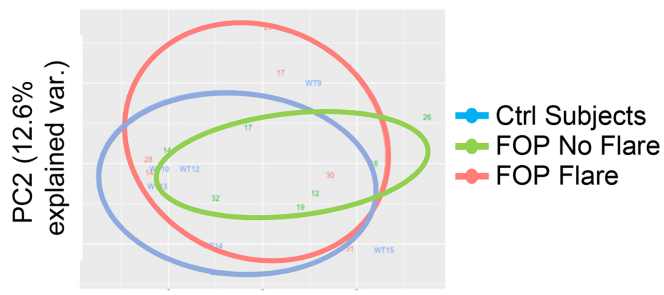

B

PC1 (31.9\% explained var.)

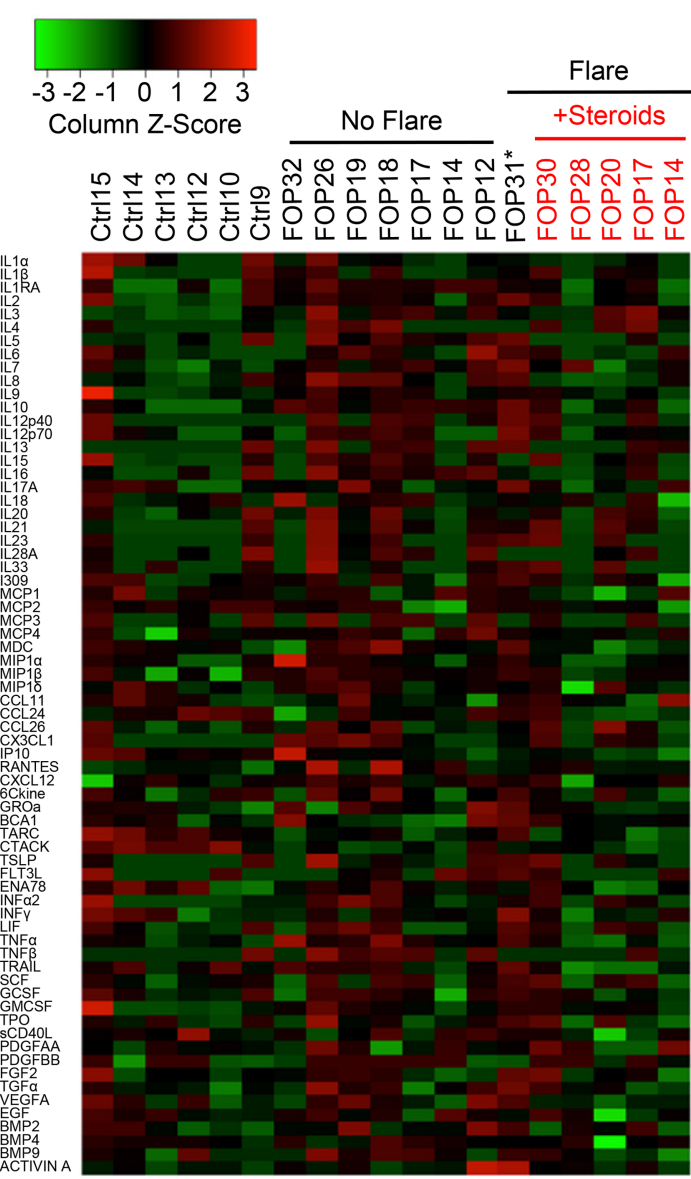

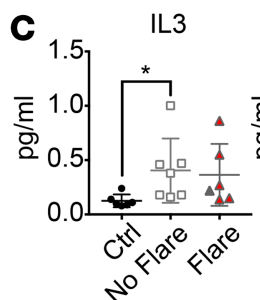

IL10

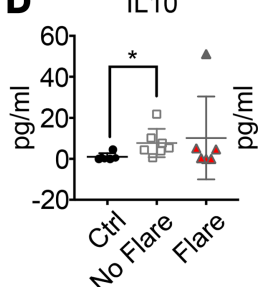

E

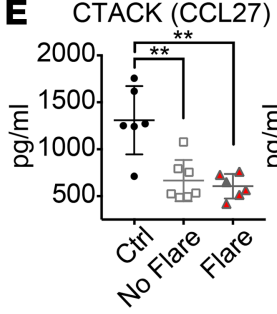

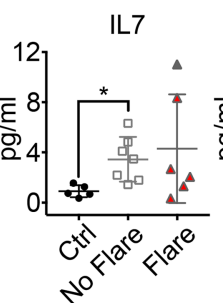

IP10 (CXCL10)

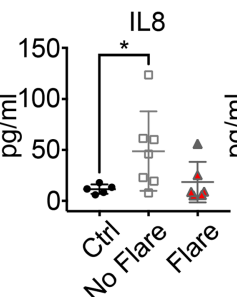

Fractalkine

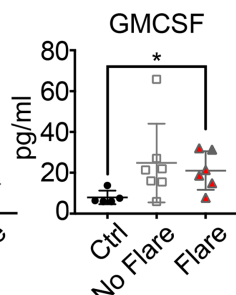

TNF-a

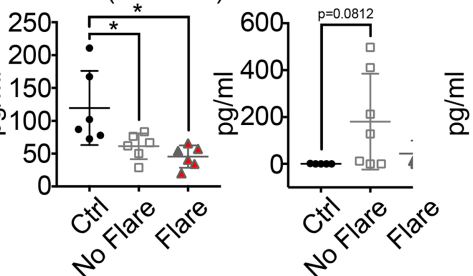

TARC (CCL17)
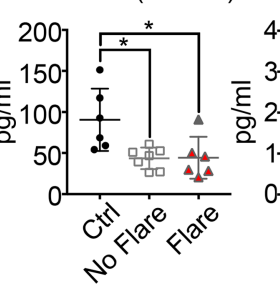

IL9

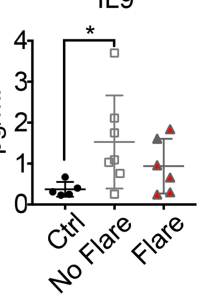

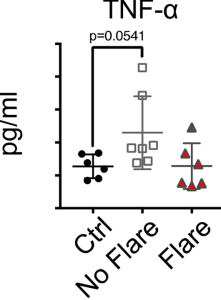

- Ctrl Subjects

$\square$ FOP No Flare

$\triangle$ FOP Flare

$\Delta$ FOP Flare+ Steroids
Figure 2. Sera of FOP patients show elevated proinflammatory cytokines. Multiplex assays for 69 cytokines on serum of control $(n=6)$, FOP subjects with clinical documented flare $(n=6)$, and FOP subjects without a flare $(n=7)$. (A) Principle component analysis of the 69 cytokines. (B) Heatmap representing color-coded cytokines/chemokines detected in the serum of control and FOP subjects, showing that FOP patients without a flare tend to show increased cytokine levels. Asterisk indicates that FOP31 was not taking steroids at the time of collection. (C and D) Myeloid and proinflammatory cytokine were significantly increased in FOP subjects with no flare. (E) Lymphoid cytokines CTACK (cutaneous T cell-attracting chemokine) and TARC (thymus and activation-regulated chemokine) were significantly lower in all FOP subjects. IL-9, which can be secreted by lymphocytes and mast cells, was elevated in FOP subjects with no flare. The distribution of the subjects is described in Supplemental Table 1. Error bars represent mean $\pm 1 \mathrm{SD}$. ${ }^{*} P<0.05,{ }^{*} P<0.01$, by Student's $t$ test.

Flare+Steroids groups showed qualitative differences when viewed on a heatmap (Figure 2B). Levels of IL-3, a critical cytokine for the maturation of the myeloid lineages (37); IL-7, important for the maturation of lymphoid lineages (37) and myeloid cell migration (38); and IL-8 produced by monocytes, macrophages, and endothelial cells (39-41) were significantly increased in sera of FOP subjects with no flare as compared with controls (Figure 2C). Granulocyte-macrophage CSF (GMCSF), a growth factor that can stimulate the differentiation of hematopoietic stem cells into granulocytes and monocytes (42), showed a trend toward increased levels in FOP subjects at baseline but was significantly increased in the serum of FOP Flare+Steroids subjects (Figure 2C). The elevation of these cytokines in sera of FOP subjects suggested that immune cells may be activated even at baseline, when no clinically active bone formation is evident in FOP subjects, and that steroids can partially buffer these cytokine changes.

We also found that levels of IL-10, an antiinflammatory cytokine (43), were significantly increased in sera of FOP No Flare subjects compared with controls (Figure 2D). IP10, which can be expressed by monocytes upon IFN- $\gamma$ stimulation (44), was significantly decreased in FOP subjects (Figure 2D). There was also a trend toward increased proinflammatory cytokines TNF- $\alpha$ and Fractalkine/CX3CL1 (a chemoattractant for monocytes and T cells involved in osteoarthritis and rheumatoid arthritis; refs. 45-49) in FOP subjects with no flare (Figure 2D). We found no differences in serum levels of BMP2, BMP4, BMP9, or Activin A (Supplemental Figure 1). Interestingly, CCL27 (cutaneous T cell-attracting chemokine [CTACK]), a skin-specific chemokine 
in wound healing, and CCL17 [thymus and activation-regulated chemokine [TARC]), a chemotactic mediator for TH2 cells, were significantly decreased in FOP subjects regardless of flare status (Figure 2E). IL-9, secreted by lymphocytes and mast cells (50), was significantly increased in FOP subjects with no flare (Figure 2E). Notably, the 1 subject (FOP31) with a FOP flare who did not take steroids (Figure 2, C-E) showed cytokine levels that tended to be outside of the \pm 1 SD range. When FOP31 was excluded from analysis, only TNF- $\alpha$, TNF-related apoptosis-inducing ligand (TRAIL), and MIP1- $\alpha$ levels became significantly different between the FOP No Flare and FOP Flare groups (Supplemental Figure 2). This suggested that steroid treatment may not affect other cytokines in FOP subjects.

These results suggested that cytokine changes are present in FOP subjects at baseline in a nonflare state and that some cytokine changes appear normalized in FOP subjects receiving standard-of-care high-dose steroids for their FOP flares. In addition, the cytokines' elevation known to stimulate monocyte recruitment, activation, and/or differentiation suggested a role for myeloid cells in human FOP.

Circulating proinflammatory monocytes are increased in FOP patients in the absence of a flare. We found no gross abnormalities in FOP No Flare subjects' peripheral blood counts (Supplemental Table 2). However, our finding of increased serum myeloid-related cytokines (Figure 2) and prior observations of abundant macrophages in developing FOP lesions (34) led us to examine if monocyte subtypes in the peripheral blood might differ between FOP and control subjects.

Using a new cohort of FOP patients and controls (Supplemental Table 3), whole blood from control, FOP subjects with no flare, and FOP subjects with a flare with or without steroids were analyzed by flow cytometry. We used exclusion/inclusion gating (Figure 3A) to identify the peripheral monocytes subsets: classical $\left(\mathrm{CD} 14^{+} \mathrm{CD} 16^{-}\right)$, intermediate $\left(\mathrm{CD} 14^{+} \mathrm{CD} 16^{+}\right)$, and nonclassical $\left(\mathrm{CD} 14^{\mathrm{lo}} \mathrm{CD} 16^{+}\right)(51)$. B and $\mathrm{T}$ lymphocytes, neutrophils, and NK cells were excluded $(51,52)$. $\mathrm{CD} 14^{+} \mathrm{CD} 16^{-}$monocytes were significantly increased in FOP subjects with a flare compared with controls. Total circulating monocytes were not different between control and FOP (Figure 3B). However, the $\mathrm{CD} 14^{+} \mathrm{CD} 16^{+}$monocyte subpopulation, which is believed to be more inflammatory (53), was significantly increased in FOP subjects with No Flare compared to control subjects (Figure $3 \mathrm{C}$ ). CD $14^{\mathrm{lo}} \mathrm{CD} 16^{+}$monocytes ("patrolling monocytes") were significantly decreased in FOP subjects with flare compared with controls subjects (Figure 3C). In addition, we didn't find any significant differences in $\mathrm{CD}^{+} \mathrm{T}$ lymphocytes (Supplemental Figure 3). These results suggested that some FOP subjects not experiencing a clinically obvious flare may have a cellular proinflammatory state at baseline. Although changes in blood monocyte counts may be related to steroid usage in the FOP Flare+Steroids group (54), these subjects were generally the lower values in the cell analyses and, thus, likely decreased significance of our assays. In addition, the changes in the FOP No Flare group are less likely to be affected due to their longer duration off of steroids.

FOP circulating monocytes subsets express higher levels of chemotaxis receptors. Since we found differences in $\mathrm{CD} 14^{+} \mathrm{CD} 16^{+}$and $\mathrm{CD} 14^{1 \circ} \mathrm{CD} 16^{+}$subsets of monocytes between control and FOP subjects, we investigated if these subtypes had different gene expression signatures. Statistical analyses of the FOP Flare and FOP No Flare groups were combined, since the groups showed no obvious differences (Figure 3, D and E). We sorted the 3 different monocyte subsets to high purity and investigated their gene expression profile. As expected, CD14, CCR2, and CD163 were highly expressed in CD14 ${ }^{+} \mathrm{CD} 16^{-}$monocytes (53) (Figure 3D). CD14 CD16 monocytes expressed significantly higher levels of the Activin A subunit inhibin $\beta$ A (INHBA) than any of the other subsets (Figure 3D), with no differences based on FOP genetic status. BMP4 and BMP6 were expressed at detectable levels in monocytes (Supplemental Figure 4), whereas BMP2 and BMP7 were not expressed at detectable levels (data not shown) in any subtypes. In addition, FOP monocytes didn't show any increase in Oncostatin M, recently shown to contribute to human neurogenic heterotopic ossifications (55) (Supplemental Figure 4). Since TLR expression was reported to be increased in FOP connective tissue progenitor cells (56), we investigated the expression of TLR2/4. Neither TLR2 nor TLR4 was increased in FOP monocytes (Figure 3E). CXCR4 (a receptor involved in stem cell homing) and activator protein 1 (AP1), which is activated by MAPKs (57), gene expression levels were significantly increased in FOP CD $14^{+} \mathrm{CD} 16^{-}$monocytes compared with control (Figure 3E). Since CXCR4 can identify transitional BM premonocytes that replenish the mature monocyte pool for peripheral responses (58), these results suggest that circulating FOP monocytes may be enriched in a transitional BM premonocyte population and may be more sensitive to immune cell homing signals.

Primary FOP monocytes show increased response to LPS compared with controls. We next asked if the ACVR1 R206H mutation might confer increased baseline monocyte activity or responsiveness to known monocyte stimuli. CD $14^{+}$monocytes were isolated from peripheral blood of control and FOP subjects 
A

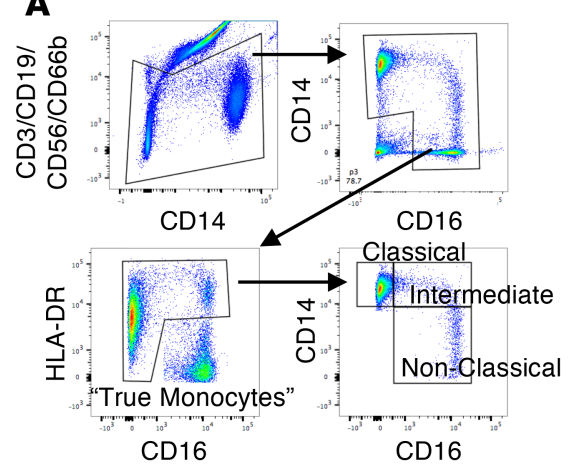

B

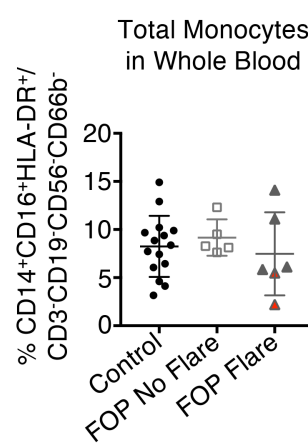

C

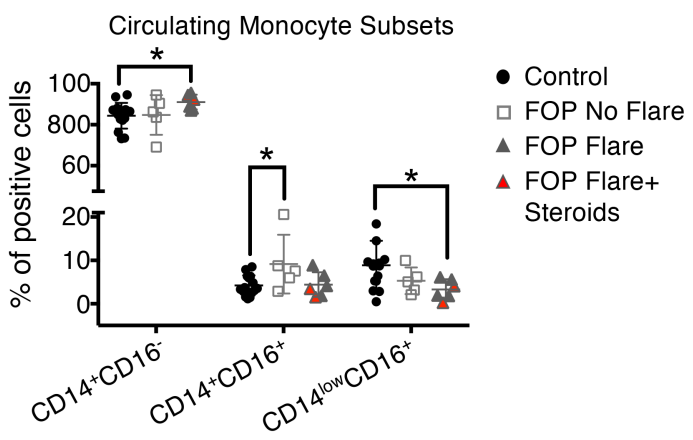

D

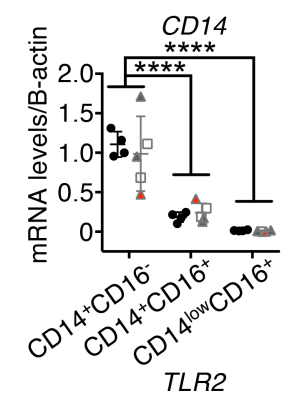

E

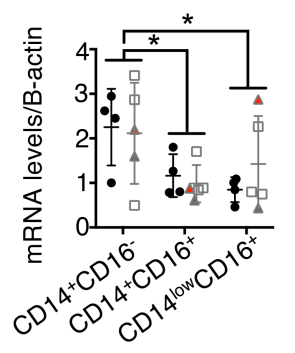

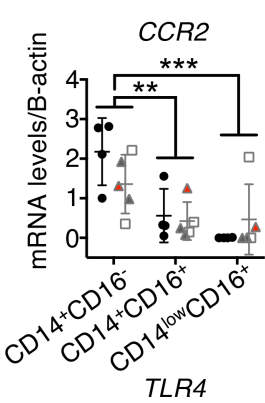

TLR4

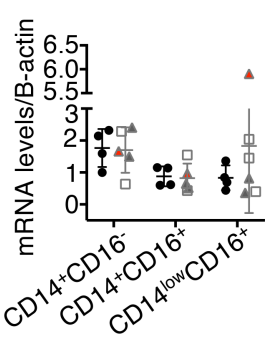

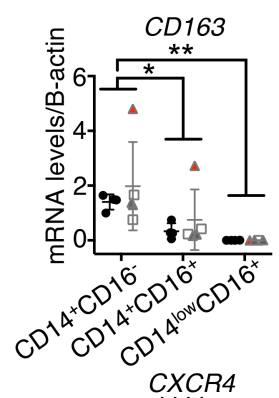

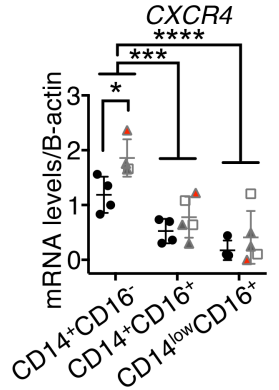

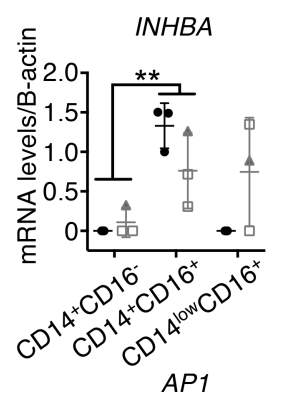

- Control

- FOP No Flare

$\triangle$ FOP Flare

$\triangle$ FOP Flare+ Steroids

Figure 3. Increased proinflammatory monocyte subsets in FOP subjects with no clinically ongoing flare. Whole blood samples from control and FOP subjects were collected, analyzed, and sorted via flow cytometry. (A) The gating strategy for identification of monocytes. (B) Total monocytes in the whole blood were not different between control and FOP subjects. (C) The intermediate monocytes (CD14 $\left.4^{+} / \mathrm{CD}_{16}{ }^{+}\right)$were significantly increased in FOP subjects with no flare. The nonclassical monocytes $\left(\mathrm{CD} 14^{+} / \mathrm{CD} 16^{+}\right)$were significantly decreased in all FOP subjects. Supplemental Table 3 shows the distribution of the subjects (Control, $n=15$; No Flare, $n=5$; Flare, $n=6$ ). ${ }^{*} P<0.05$, by Student's $t$ test. (D) Monocyte subtypes were sorted, and RNA was extracted. Gene expression analysis of monocyte receptors. CD14, CCR2, and CD163 were decreased in the CD14+CD16 and CD14 ${ }^{\circ} \mathrm{CD} 16^{+}$population in both control and FOP monocytes. INHBA (a subunit of Activin A) was significantly increased in the CD14+CD16+ monocytes of both control and FOP. (E) TLR2 and TLR4 were not increased in FOP monocytes, while CXCR4, a potential marker of premature monocytes, and $A P 1$ were significantly increased in FOP CD14 ${ }^{+} C D 16^{-}$monocytes compared with control. Supplemental Table 4 shows the distribution of the subjects (Control, $n=4 ; \mathrm{FOP}, n \geq 3$ ). ${ }^{*} P<0.05,{ }^{* *} P<0.01$, by 2 -way ANOVA Sidak or Tukey's multiple comparison test. Error bars represent mean \pm 1 SD.

(Supplemental Table 5 and Figure 4A). Expression of typical extracellular monocytes markers (CD14, CD11B, CD163, and CD206) were not significantly different between groups (Figure 4B).

Control and FOP primary monocytes were stimulated with different concentration of gram-negative bacterial LPS, a potent TLR4 signaling activator (59). FOP CD14 $4^{+}$primary monocytes showed significantly increased CCL5, CCR7, and CXCL10 expression when stimulated with $10 \mathrm{ng} / \mathrm{ml}$ LPS (Figure 4C). These genes are typically expressed in polarized monocytes/macrophages (60) and are proinflammatory. ACVRI is expressed in monocytes (Figure 4C). We found no significant differences between $100 \mathrm{ng} / \mathrm{ml}$ LPS-stimulated control and FOP monocytes (Figure 4C). In addition, inhibitor of DNA binding 1 (IDI) expression, a direct BMP pathway target (61), was decreased upon LPS stimulation, suggesting that the BMP pathway may not be dominant in the monocyte response to LPS (62) (Figure 4D). All LPS-stimulated monocytes showed increased Activin A gene expression, but we found no significant differences between control and FOP monocytes (Figure 4D). Also, LPS-stimulated FOP monocytes did not show increased TLR2 and $T L R 4$ expression (Figure 4D), as previously described in connective tissue progenitor cells (56), suggesting that the increased LPS response in FOP monocytes was not due to increased TLR expression. Finally, FOP CD14 ${ }^{+}$monocytes stimulated with TLR2 ligands P3C (Pam3CSK4, synthetic triacylated lipoprotein) 
A

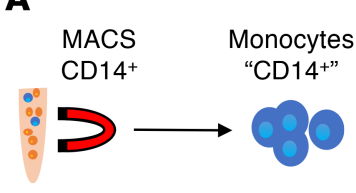

C
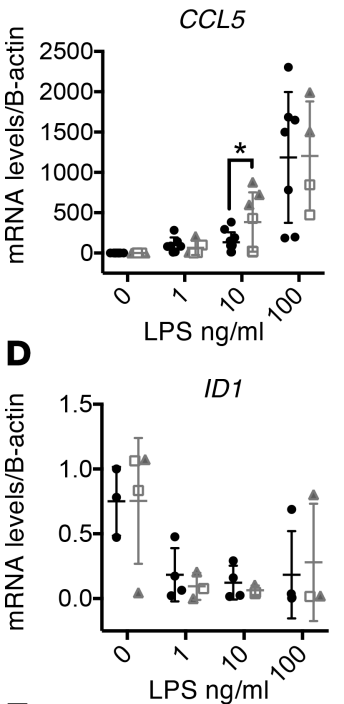

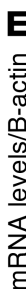

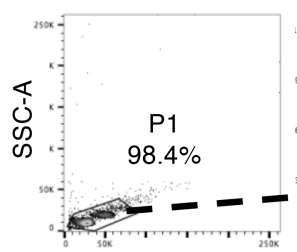

FSC-A
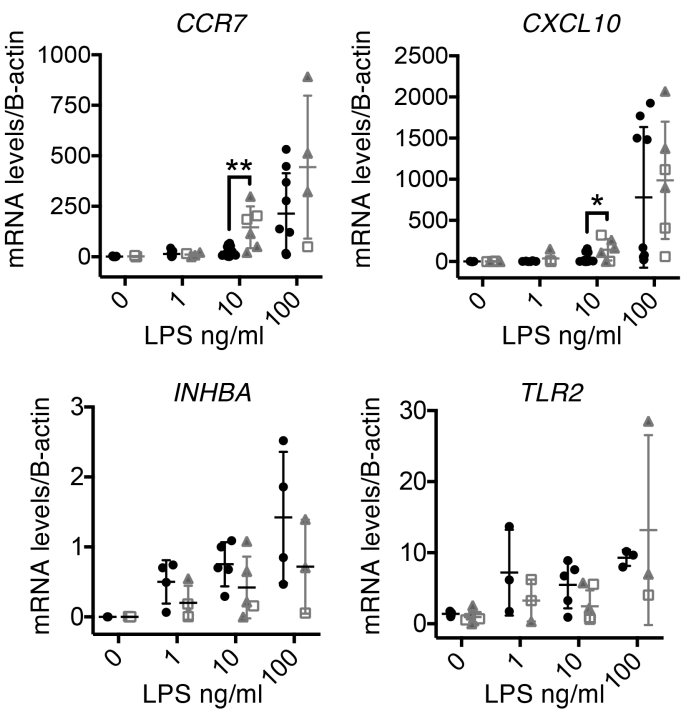

CXCL10

B
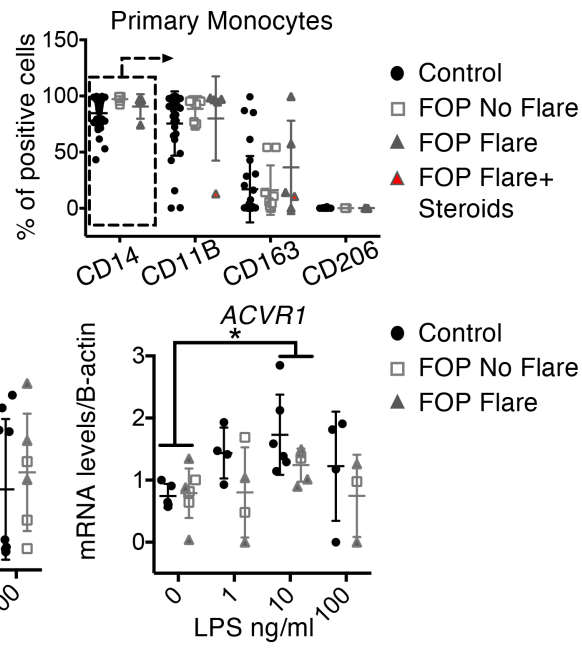

- Control

$\square$ FOP No Flare

$\Delta$ FOP Flare
- Control

$\triangle$ FOP Flare $\square$ FOP No Flare
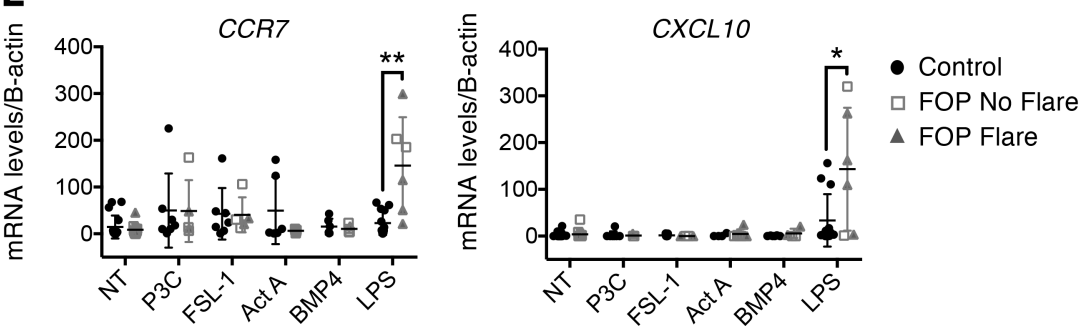

Figure 4. Increased response to LPS by primary FOP monocytes. (A) Primary monocyte FOP subjects were purified via MACS for CD14. (B) CD14+ cells (in rectangle) from control or FOP subjects showed no significant differences in CD11B, CD163, or CD206 expression. (C) FOP CD14 ${ }^{+}$monocytes showed a significant increase in gene expression of CCL5, CCR7, and CXCL10 at lower LPS concentration. (D) SMAD1/5-driven expression of ID1 (inhibitor of DNA binding 1) was not increased in FOP monocytes. INHBA was substantially increased in all monocytes when stimulated with increasing amounts of LPS. The increased LPS response was not due to an increase in TLR receptor expression. (E) Control and FOP monocytes were stimulated with TLR2 ligands, BMP4, or Activin A. Control and LPS samples from $\mathbf{C}$ were presented again in $\mathbf{E}$ for comparison with other ligands. The distribution of the subjects is described in Supplemental Table 5 (control, $n \geq 3$; FOP, $n \geq 3$ ). ${ }^{*} P<0.05,{ }^{* *} P<0.01$, by multiple comparison Student's $t$ test. Error bars represent mean \pm SD.

or FSL1 (synthetic ligand derived from mycoplasma salivarium), or with Activin A or BMP4, did not show significant differences in CCR7 or CXCL10 levels (Figure 4E). Together, these results suggest that the ACVR1 R206H mutation may induce increased responsiveness to TLR4 ligands. In addition, monocytes can increase their Activin A production in response to LPS, but FOP monocytes do not appear to produce significantly more Activin A than controls.

Stimulated FOP monocytes show abnormal cytokine/chemokine secretion. Since LPS appeared to induce a shift in the response curve of FOP peripheral monocytes, we examined the functional activity of cultured LPS-stimulated monocytes using a multiplex assay for 41 cytokines on culture supernatant (Figure 5, A and B, and Supplemental Table 6). PCA analysis of the cytokines/chemokines showed that LPS-stimulated and LPS-unstimulated FOP monocytes clustered separately from control monocytes (Figure 5A). Proinflammatory cytokines, including IL-1 receptor antagonist (IL-1RA), IL-3, IL-15, and IL-17A in FOP monocytes after LPS stimulation (Figure 5C), were significantly increased. IL-1 $\alpha$, IL-1 $\beta$, IL-6, and TNF- $\alpha$ levels were increased in LPS-stimulated control and FOP monocytes, but there were no differences between control and FOP LPS-stimulated monocytes (Figure 5D). MIP1- $\alpha$ and eotaxin (CCL11) were significantly elevated in LPS-treated FOP monocytes compared with control (Figure 5E). Finally, EGF and VEGFA 
A

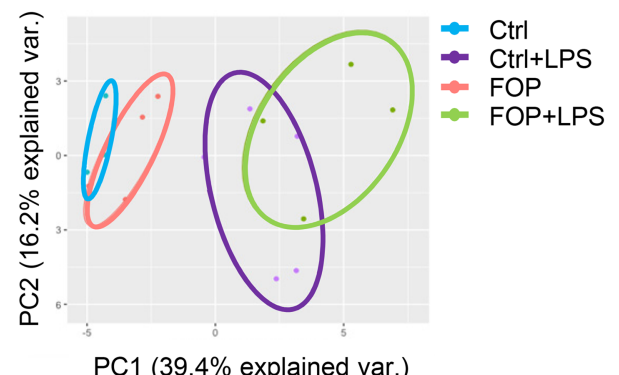

PC1 (39.4\% explained var.)

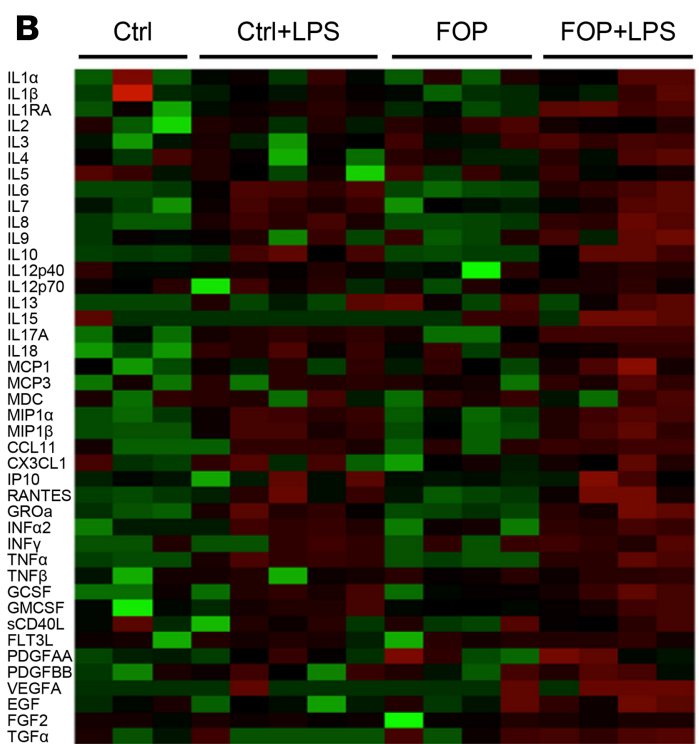

C

IL1RA

IL3

IL15

IL17A

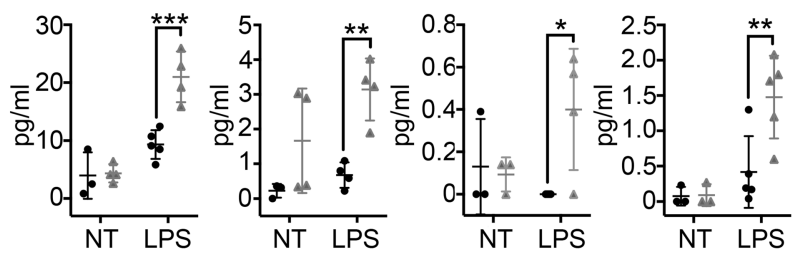

IL1a

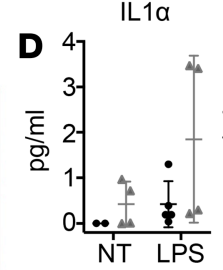

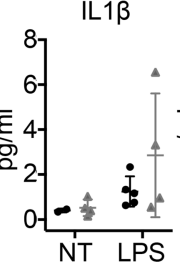

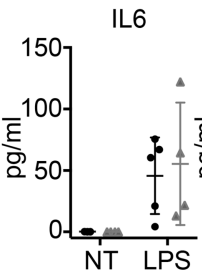

1500-

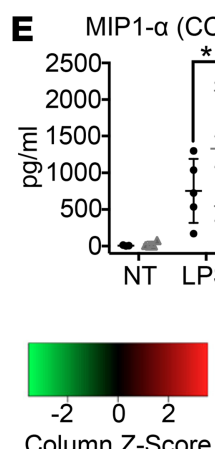

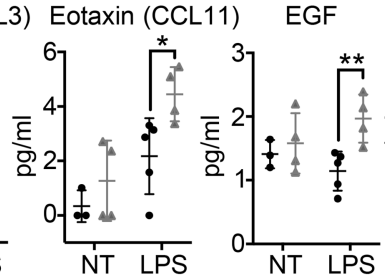

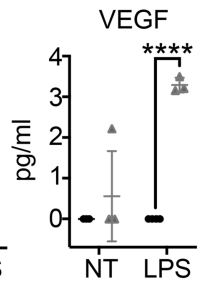

- Control

$\triangle$ FOP Flare

Figure 5. LPS-stimulated FOP monocytes show abnormal proinflammatory cytokine secretion. Supernatants were collected from monocytes of control $(n \geq 3)$ and FOP $(n=4)$ subjects. Monocytes untreated (NT) and LPS-stimulated (10 ng/ml) for 24 hours were assayed for 41 cytokines. (A) Shapes represent expression profiles of the different donors in principle component analysis (PCA). (B) Heatmap representing color-coded cytokine secretion by Control, Control+LPS, FOP, FOP+LPS samples. (C) Proinflammatory cytokines IL-1RA, IL-3, IL-15, and IL-17A were significantly increased in FOP LPS-stimulated monocytes. (D) Typical proinflammatory cytokines IL-1 $\alpha / \beta$, IL-6, and TNF- $\alpha$ production were increased upon LPS stimulation but not significantly different among control and FOP subjects. (E) Chemokine ligands CCL3 and CCL11 and growth factors EGF and VEGF were significantly increased in FOP monocytes stimulated with LPS. The distribution of the subjects is described in Supplemental Table $6 .{ }^{*} P<0.05,{ }^{* *} P<0.01,{ }^{* * *} P<0.001$ by Sidak's multiple comparison test. Error bars represent means $\pm 1 \mathrm{SD}$.

growth factors were significantly increased in LPS-stimulated FOP monocytes (Figure 5E). Although both EGF and VEGFA can mediate monocyte chemotaxis (63-65), secretion of these 2 growth factors may favor the recruitment of angiogenic cells $(66,67)$. Together, these results suggested that primary monocytes from FOP subjects have an abnormal response to TLR4 stimulation by LPS that leads to secretion of elevated proinflammatory cytokines, chemokines, and growth factors.

Prolonged activation of NF- $\kappa B$ signaling pathway in LPS-stimulated FOP monocytes. The inflammatory and SMAD signaling pathways can converge via TGF- $\beta$ activated kinase 1 (TAK1) $(68,69)$. Thus, we asked if the increased LPS responses observed in FOP monocytes might be due to a TAK1 dysregulation. TAK1 phosphorylation was increased upon LPS stimulation in control and FOP monocytes (Figure 6, A and B). Expression was highly variable within the FOP monocytes. No significant differences were found when TAK1 phosphorylation was normalized to $\beta$-actin. However, when normalized to total TAK1, both control and FOP monocytes showed increased TAK1 phosphorylation after 30 minutes of LPS stimulation, with a trend toward higher levels in FOP monocytes (Figure 6B). These results hint that TAK1 dysregulation may be present in FOP monocytes, but further experiments using larger numbers of cells are needed to understand the role of TAK1 in FOP.

We next asked if the inflammatory (NF-kB vs. MAPKs) and SMAD pathways might be active in FOP monocytes as a potential cause of the abnormal cytokine secretion we observed. Surprisingly, SMAD1/5/9 phosphorylation and NF- $\mathrm{\kappa B}$ total lysate phosphorylations were not significantly different between control and FOP monocytes (Figure 6, A and B). Since we had limited monocytes obtainable 
A
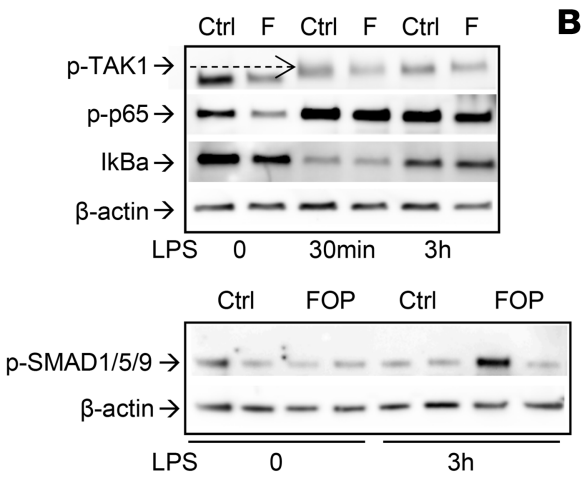

C

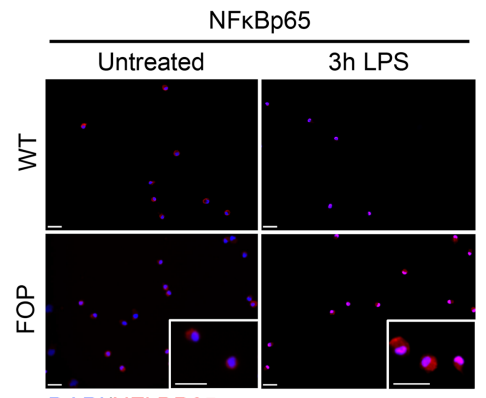

D

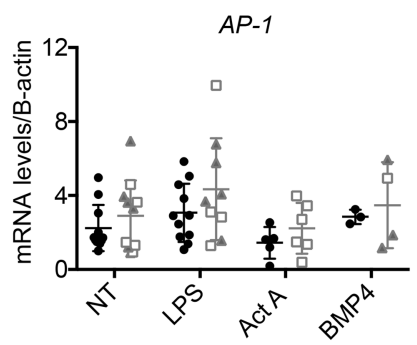

B

p-TAK1/b-actin

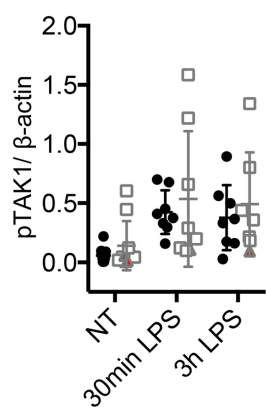

p-TAK1/t-TAK1

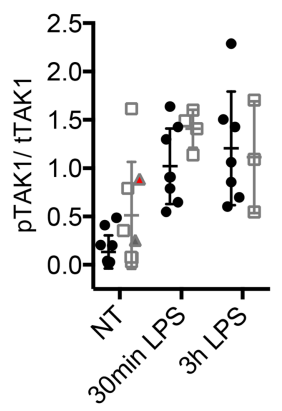

Total lysate p-NFkBp65

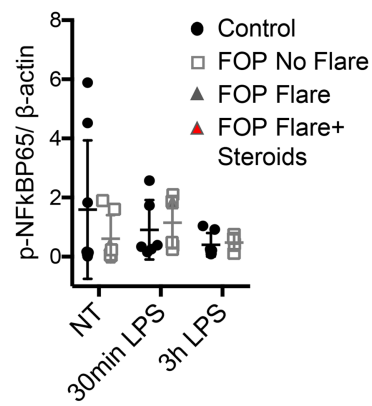

Nuclear NFkBp65
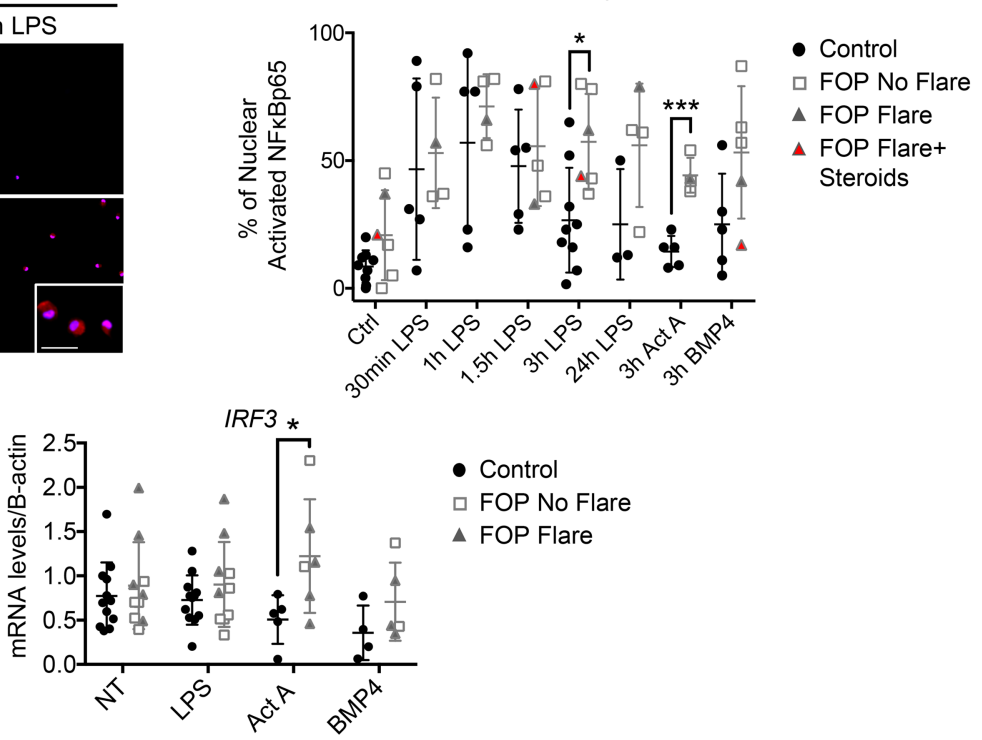

- Control

$\square$ FOP No Flare

$\square$ FOP No Fla
$\triangle$ FOP Flare

Figure 6. Increased NF-кB activity in FOP monocytes. (A) Representative Western blots (repeated for at least 3 different biological samples) showing activation of TCF- $\beta$ activated kinase 1 (TAK1) and NF- $\mathrm{KBp} 65$ phosphorylation, as well as IkBa degradation (top) in control and FOP monocytes. The SMAD1/5/9 pathway is not activated in FOP monocytes (bottom). (B) TAK1 phosphorylation is increased upon LPS stimulation (10 ng/ml) in both control $(n \geq 5)$ and FOP $(n \geq 4)$ monocytes. There were no significant differences in total lysate NF- $\kappa$ Bp65 phosphorylation upon LPS stimulation. (C) Time course of LPS-induced NF- $\mathrm{KBp} 65$ nuclear translocation in cells stimulated with LPS (10 ng/ml), BMP4 (50 ng/ml), or Activin A (50 ng/ml). Immunofluorescence staining of NF- $\kappa$ Bp65 and quantification of nuclear staining (NF- $\kappa$ Bp65:Dapi) shows a significant increase of NF- $\kappa$ Bp65 nuclear translocation in FOP monocytes when stimulated with LPS and Activin A for 3 hours (control, $n \geq 3$; FOP, $n \geq 4$ ). Scale bars: $50 \mu \mathrm{m}$. ${ }^{*} P<0.05$ and ${ }^{* * *} P$ $<0.005$ by multiple comparison Student's $t$ test. Error bars represent means $\pm 1 \mathrm{SD}$. At least 100 nuclei were evaluated per condition. (D) mRNA levels of IRF3 were increased with Activin A stimulation in FOP monocyte (control, $n \geq 3 ;$ FOP, $n \geq 3$ ). ${ }^{*} P<0.05$, by multiple comparison Student's $t$ test. Error bars represent mean \pm 1 SD. The distribution of the subjects is described in Supplemental Tables 7 and 8 .

from FOP subjects, we used immunofluorescence staining to semiquantitatively assess the NF- $\mathrm{Bp} 65$ (Figure 6C), phospho-p42/44 (ERK1/2), p38, and phospho-SMAD1/5 translocation from the cytoplasm to the nucleus (Supplemental Figure 5).

Control and FOP monocytes were stimulated with LPS, Activin A, or BMP4. NF-кBp65 nuclear translocation percentage decreased after 1.5 hours of LPS stimulation in control monocytes, while

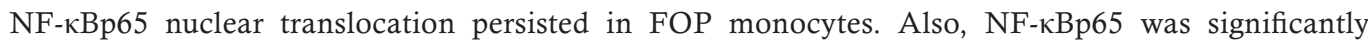
increased in Activin A-stimulated FOP monocytes (Figure 6C). Phospho-p42/44 showed a trend toward elevation in FOP monocytes (Supplemental Figure 5). Nuclear-activated p42/44 percentage was significantly increased in BMP4-stimulated FOP monocytes. Nuclear-activated p38 and phospho-SMAD1/5 were not significantly different between control and FOP monocytes, even when stimulated with Activin A or BMP4 (Supplemental Figure 5). AP1, a transcription factor activated by MAPKs and NF- $\mathrm{B}$ pathways, showed no significant differences between control and FOP monocytes. However, IFN regulatory factor 3 (IRF3) was significantly increased in FOP monocytes stimulated with Activin A (Figure 6D). 
A

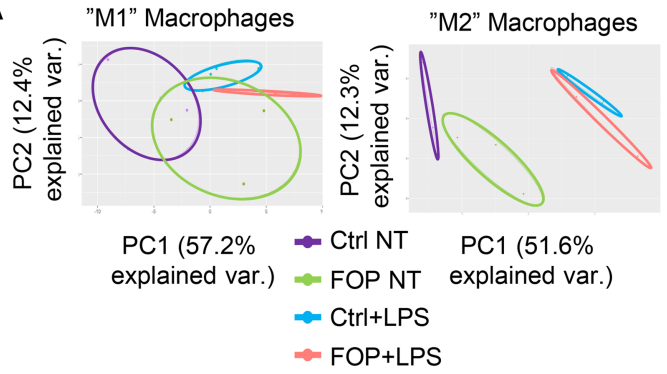

B

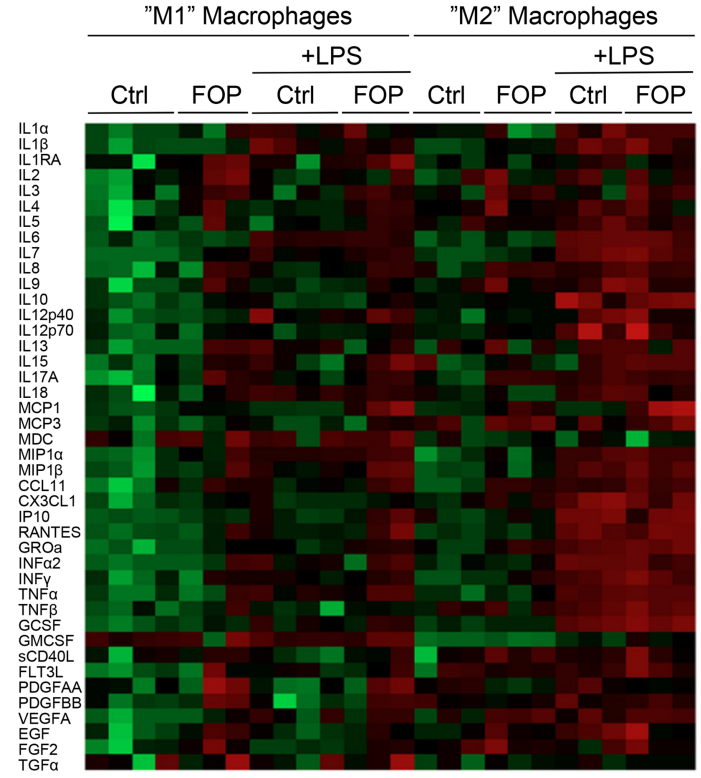

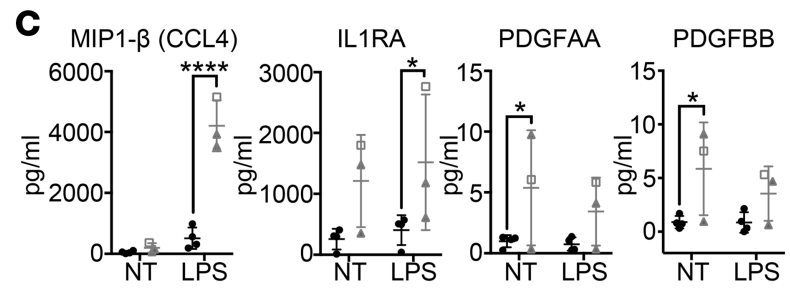

- Control

口 "M1" FOP No Flare

^ "M1" FOP Flare

D

Eotaxin (CCL11) VEGF

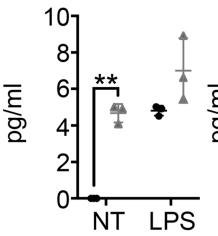

- Control

口 "M2" FOP No Flare

4 "M2" FOP Flare

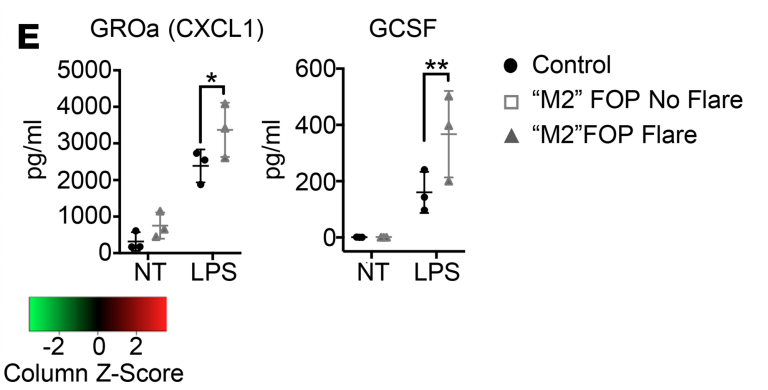

Figure 7. LPS-stimulated FOP macrophages show abnormal proinflammatory cytokine secretion. Primary CD14 ${ }^{+}$monocytes were differentiated into proinflammatory (stimulated with GMCSF, M1) or antiinflammatory (stimulated with MCSF, M2) macrophages. Multiplex assay for 41 cytokines on M1 (control, $n=4$; FOP, $n=3$ ) and M2 (control, $n=3$; FOP, $n=3$ ) macrophages were either untreated (NT) or treated with $10 \mathrm{ng} / \mathrm{ml}$ of LPS for 24 hours. (A) Principle component analysis. Shapes represent expression profiles of the different groups. (B) Heatmap representing color-coded cytokine secretion by Control, Control+LPS, FOP, FOP+LPS for M1 and M2 macrophage samples. (C) Cytokines MIP1- $\beta$ and IL-1RA were significantly increased in FOP M1 polarized macrophages stimulated with LPS. PDGFAA and PDGFBB were significantly increased in nonstimulated FOP M1 polarized macrophages. (D) Chemokine ligands eotaxin, VEGF, IL-3, and IL-17A were increased at baseline in FOP subject M2 macrophages. (E) GRO $\alpha$ and GCSF were significantly increased in FOP monocytes stimulated with LPS. The distribution of the subjects is described in Supplemental Table $9 .{ }^{*} P<0.05,{ }^{* *} P<0.01$, and ${ }^{* * * *} P<0.0001$ by Sidak's multiple comparison test. Error bars represent means $\pm 1 \mathrm{SD}$.

These results indicate that the abnormal cytokine secretion observed in FOP monocytes is likely due to

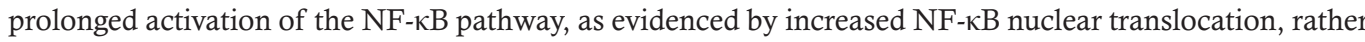
than increased SMAD1/5 activity.

FOP primary monocyte-derived macrophages also show abnormal cytokine/chemokine secretion. Since FOP monocytes showed increased responsiveness to LPS, we asked if primary FOP monocyte-derived macrophages responded similarly. $\mathrm{CD} 14^{+}$monocytes isolated from peripheral blood of control and FOP subjects were stimulated with GMCSF for 7 days to polarize toward a proinflammatory (M1) phenotype, or MCSF (macrophage CSF) to polarize toward an antiinflammatory (M2) phenotype. We investigated their response to LPS using the same multiplex assay previously used for monocytes (Figure 5). PCA and heatmap analyses of the 41 cytokines (Figure 7, A and B, and Supplemental Table 9) for the proinflammatory M1 macrophages (Figure 7A, left) showed that LPS-stimulated and LPS-unstimulated FOP subjects clustered separately from the control subjects. Surprisingly, antiinflammatory M2 macrophages from unstimulated FOP subjects clustered even further away from control subjects (Figure 7A, right). Proinflammatory cytokines in FOP proinflammatory M1 macrophages (GMCSF stimulated), including MIP1- $\beta$, IL-1RA, PDGFAA, and PDGFBB (Figure 7C), were significantly increased. Cytokines IL-3 and IL-17A and chemokines eotaxin and growth factor VEGFA were significantly increased in FOP antiinflammatory M2 macrophages (stimulated with MCSF) in a non-LPS-stimulated state 
A
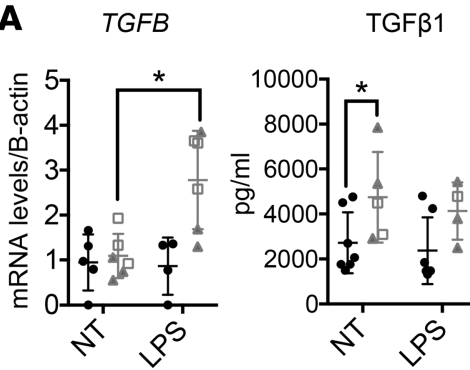

- Control "M2"

- FOP No Flare "M2"

\ FOP Flare "M2"

\section{C}

$\frac{\text { "M1" Macrophages (GMSCF stimulated) }}{\text { p-p38MAPK } \quad \text { p-NFKBp65 }}$
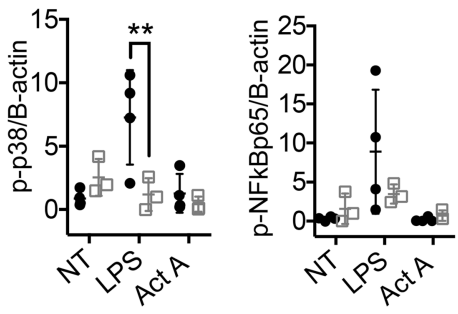

- Control

- FOP No Flare

$\triangle$ FOP Flare

$\triangle$ FOP Flare+

Steroids

"M2" Macrophages (MCSF stimulated)

p-p38MAPK

p-NFKBp65
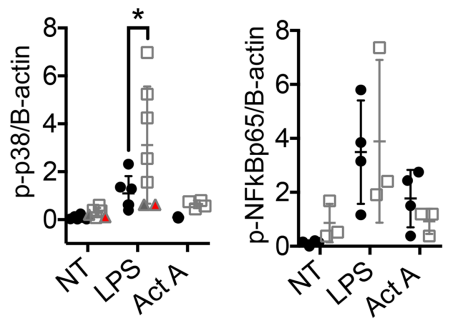

- Control

- FOP No Flare

$\triangle$ FOP Flare

^ FOP Flare+

Steroids

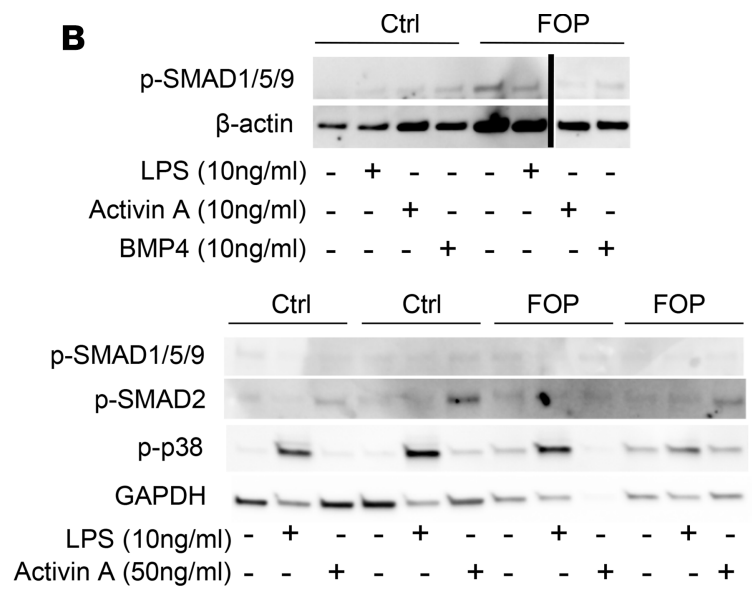

D
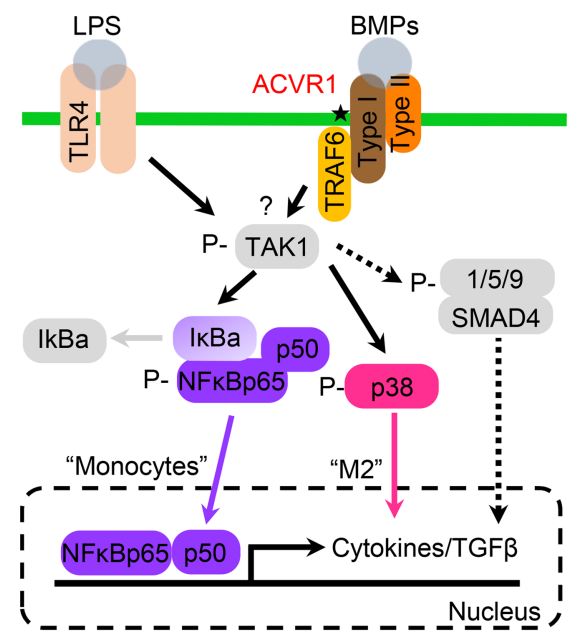

Figure 8. p38MAPK pathway is dysregulated antiinflammatory macrophages. (A) Control $(n \geq 4)$ and FOP $(n=6)$ macrophages were stimulated with LPS. TCFB gene expression was significantly increased in LPS-stimulated FOP M2 macrophages. TGF- $\beta 1$ ELISA on cell supernatant revealed a significant increase in FOP M2 macrophages. (B) Representative Western blots (conducted on 2 different biological samples for control and FOP) showing SMAD1/5/9 pathway is not activated in FOP macrophages upon LPS, Activin A, or BMP stimulation (top). SMAD2 phosphorylation is not upregulated in FOP macrophages upon Activin A stimulation (bottom). (C) Quantification of p38 and NF- $\kappa B p 65$ phosphorylation of control $(n \geq 4)$ and FOP ( $n \geq 3)$ M-CSF polarized macrophages when stimulated with LPS or Activin A for 2 hours. ${ }^{*} P<0.05$ by 2 -way Anova Sidak's multiple comparison test. Error bars represent mean \pm 1 SD. The distribution of the subjects is described in Supplemental Tables 10 and 11. (D) Summary of the possible activated pathways responsible for the FOP monocyte/macrophage increased inflammatory responses.

(Figure 7D. Growth-regulated oncogene- $\alpha$ (GRO $\alpha)$ and granulocyte CSF (GSCF) were significantly increased in LPS-stimulated FOP macrophages (Figure 7E). These results suggest that both FOP proand antiinflammatory macrophages secrete increased levels of cytokines and chemokines at baseline and when stimulated with LPS.

Upregulation of the $p 38$ pathway and increased TGF- $\beta$ in M2 FOP macrophages. Our supernatant analysis showed evidence of functional differences between FOP and control macrophages. Macrophages can produce TGF- $\beta$, a secreted protein implicated in fibrosis and heterotopic ossification $(25,70)$. TGFB expression was significantly increased in FOP antiinflammatory M2 macrophages upon LPS stimulation (Figure 8A). TGF- $\beta 1$-secreted levels were also higher in unstimulated FOP antiinflammatory M2 macrophages (Figure $8 \mathrm{~A})$. We found a trend toward elevated levels of TGF- $\beta 1$ in stimulated FOP macrophages, but it was not statistically significant, likely due to biological variability. These results suggest that M2 macrophages may contribute to the fibrosis $(70,71)$ seen in developing heterotopic ossification lesions (25). Phagocytic abilities of FOP macrophages showed no major differences from control macrophages (Supplemental Figure 6).

Since we previously found increased cytokine secretion in FOP monocytes due to an upregulation of the NF- $\mathrm{kB}$ pathway, we asked if the same mechanism also occurred in FOP macrophages. CD14 ${ }^{+}$ monocytes stimulated with GMCSF (M1), or with MCSF (M2), were treated with LPS or Activin A. 


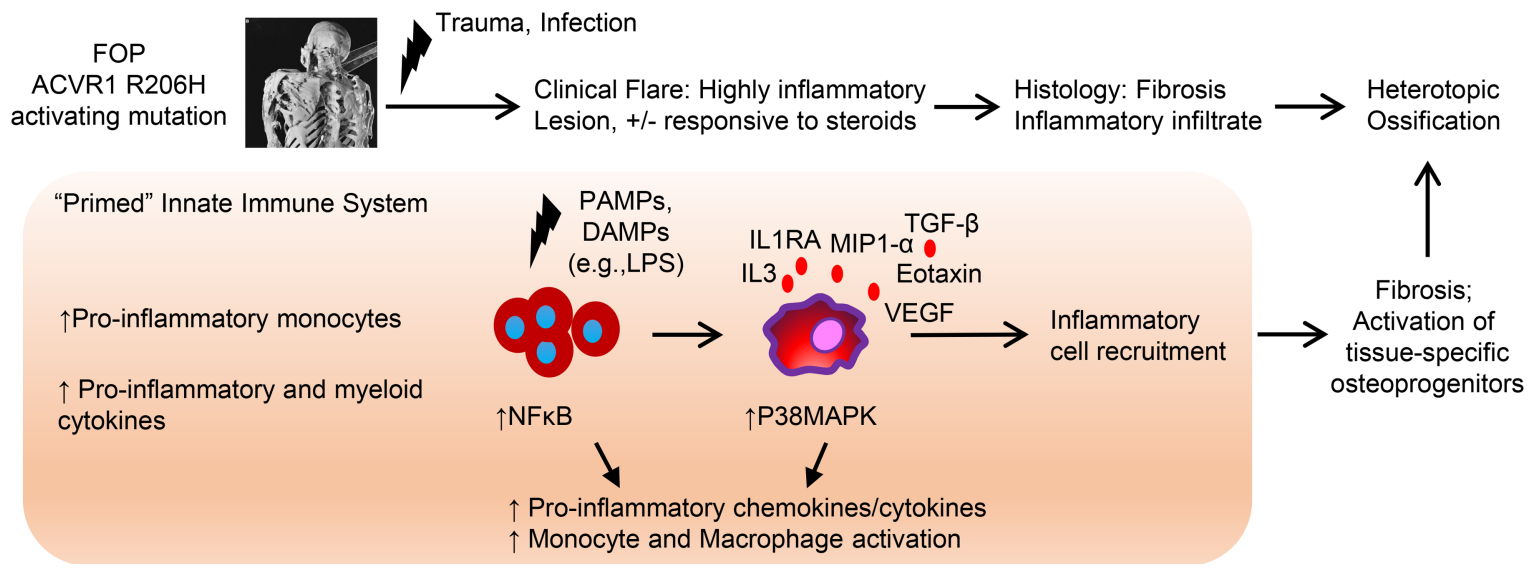

Figure 9. Summary of the innate immune system dysfunction in FOP and other forms of heterotopic ossification. FOP patients showed increases of several proinflammatory and myeloid cytokines in their serum and increased proinflammatory monocytes, suggesting a proinflammatory state at baseline. We found that FOP CD14+ monocytes and M1/M2 macrophages are hyperresponsive to TLR4 ligand LPS, which may increase and propagate the inflammatory response leading to the recruitment of other inflammatory cells and tissue-specific osteoprogenitors, which are critical steps in heterotopic bone formation.

Again, SMAD1/5/9 phosphorylation was not increased at baseline or with LPS in FOP macrophages (Figure 8B). BMP4 stimulation of control and FOP macrophages did not increase SMAD1/5/9 signaling, either (Figure 8B). The p38 pathway was significantly downregulated in FOP proinflammatory M1 macrophages stimulated with LPS, while it was elevated in LPS-stimulated FOP antiinflammatory M2 macrophages (Figure 8C). In contrast, no significant differences in NF- $\mathrm{BB}$ were identified (Figure $8 \mathrm{C}$ ). These results suggest that non-SMAD1/5/9 pathways may be critical in FOP macrophage dysfunction and that the $\mathrm{p} 38$ phosphorylation changes are most pronounced in the antiinflammatory M2 macrophage population (Figure 8D).

\section{Discussion}

Inflammation is a critical regulator of growth and repair in a wide spectrum of tissues $(1,2)$, including bone (3-8). Clinical conditions affecting inflammatory function are associated with skeletal effects, ranging from bone loss (e.g., inflammatory osteoporosis) $(72,73)$ to bone gain (heterotopic ossification) $(6,7)$. Prior studies identified numerous cytokine changes in these conditions $(18,19)$; however, the wide variety of triggers and biological responses makes it difficult to systematically elucidate the role of the immune system in skeletal diseases. In addition, while multiple cell type can clearly contribute to bone formation in heterotopic ossification (74-78), all forms of human heterotopic ossification appear to be strongly associated with inflammatory responses (7). This suggests that a common proinflammatory pathway may be a key activator of bone formation in normally nonmineralized sites.

Our systematic study showed that FOP patients have an immune system that is predisposed to over-activation, suggesting that FOP might be considered an autoinflammatory disease. We found a significant proinflammatory state at baseline in FOP patients that were not showing clinical signs of active bone formation. These changes could be detected both by multiplex serum cytokine profiling and by functional analysis of peripheral blood monocytes and macrophages. Our results showed both broad changes and focused cytokine elevations, suggesting that the ACVR1 R206H mutation may cause very specific alterations in immune function that result in global effects.

We found elevated levels of IL-9, secreted by T cells or mast cells (79), and eotaxin, a chemokine for mast cells (80), in FOP subjects with no flare and FOP monocytes/macrophages, respectively. Levels of GRO $\alpha$ were also increased in FOP monocytes/macrophages. With eotaxin, GRO $\alpha$ can promote granulocytes proliferation and differentiation at the site of injury (81-84). This may explain the large number of mast cells observed in developing FOP lesions and the mast cells' contribution to the disease's proinflammatory aspect $(24,34)$. Indeed, the depletion of mast cells and macrophages impairs heterotopic ossification formation in an ACVR1 R206H mouse model of FOP (24). Whether this occurs in humans remains unknown. Other cytokines we found elevated in FOP subjects' sera or monocytes/macrophages, such as IL-1RA (a natural IL-1 inhibitor) (85), IL-10 (43), and IL-17A (86), 
have been associated with inflammatory and autoimmune diseases. It is intriguing to consider that these autoinflammatory conditions also show intermittent flare-ups, much like what occurs in FOP.

There were some additional surprises in our results. Our serum cytokine analyses showed suppression of CTACK and TARC, 2 cytokines that may have roles in T cell function. Since T cells and macrophages can regulate each other (87), the ACVR1 R206H mutation might also affect the adaptive immune system or crosstalk between monocytes/macrophages and lymphocytes. The elevated serum IL-7, IL-8, and IL-15 can also affect maturation and activation of the lymphoid lineage $(38,88)$ and may reflect a yet-to-be identified role for $\mathrm{T}$ cells.

Several secreted factors were notably unchanged in serum, including Activin A, which is thought to act as a ligand for the ACVR1 R206H receptor $(30,31)$, and BMPs, which are thought to have enhanced activity on the ACVR1 R206H receptor (89). It is yet unknown, to our knowledge, if tissue levels of these cytokines are changed in FOP patients, but identifying sources of potential ligands for ACVR1 will be critical for developing targeted therapeutics.

Significantly elevated growth factors (PDGFs, VEGF, and TGF- $\beta$ ) and cytokines (MIP1- $\beta$, eotaxin, GRO $\alpha$ ) in FOP macrophages may also impact the differentiation or recruitment of osteoprogenitors. MIP1- $\beta$, eotaxin, and GRO $\alpha$ are upregulated during the acute phase of fracture healing (90). PDGFBB ${ }^{+}$ cells and PDGFBB in serum are increased during heterotopic ossification progression (25). PDGFs, which can be produce by macrophages (91), are chemoattractant for osteoprogenitors (92) and may recruit mesenchymal cells and osteoprogenitors to form heterotopic ossification. In addition, VEGF is essential for bone vascularization, fracture repair, and chondro- and osteogenic precursor function. Finally, the increased production of TGF- $\beta$ found in our study correlates with recent findings where TGF- $\beta$ levels are elevated in human heterotopic ossification and can trigger heterotopic ossification (25). Further investigation of whether local tissue-resident macrophages are affected by the ACVR1 R206H mutation, and whether communication with other immune cell lineages occurs, remain important challenges to overcome. While mouse models may elucidate these mechanisms, mice and humans clearly have different immune responses (93); therefore, understanding the human context using other human models will be revealing.

Critically, our results on FACS-purified FOP monocytes showed increased dose-response and hyperresponsiveness to the TLR4 ligand LPS in vitro. This shift in sensitivity correlates with clinical observations (94), suggesting that a normal immune system can still trigger FOP flares, particularly when maximally activated. Our results also suggest that ACVR1 R206H induces a cell-autonomous increase in macrophage sensitivity to activating stimuli. Although LPS is not considered to be a typical inducer of FOP flares, numerous endogenous TLR4 danger-associated molecular pattern (DAMP) proteins are induced after injury, including HMGB1, HSP, and hyaluronan, which are all crucial for tissue repair and regeneration (95). Pathogen-associated molecular patterns (PAMPs) or DAMPs released after injury may induce a hyperinflammatory response in FOP patients, causing the inflammatory soft tissue swelling during bone formation flares. The ACVR1 R206H effect in macrophages may be TLR4-pathway selective, since testing with ligands for TLR2/ 6 does not appear to stimulate increased responses. Identifying these DAMPs will be a high priority in future studies.

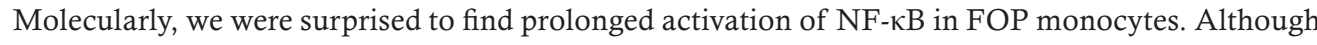
an earlier study in patient-derived lymphoblastoid cell lines suggested that NF- $\mathrm{B}$ was not significantly changed in FOP (96), TLR signaling dysregulation can occur in FOP connective tissue progenitor cells (56). NF- $\mathrm{\kappa B}$ is part of the immune defense mechanism and a master regulator of inflammation (97). Increased NF- $\kappa \mathrm{B}$ activity is sufficient to drive chronic inflammation (98) and may explain the TLR4 hyperresponsiveness we observed (99). Unexpectedly, FOP macrophages' hyperresponsiveness is likely mediated by 2 different pathways, whether they are monocytes (NF- $\mathrm{\kappa B})$ or differentiated antiinflammatory macrophages (p38). ACVR1 and TLR4 can regulate the expression of TAK1 $(69,100)$, which activates the NF- $\mathrm{KB}(97)$ and SMAD1/5 pathway $(69,100)$. Surprisingly, SMAD1/5/9 signaling was not changed in FOP monocyte/macrophages. This suggests that tissue-specific activation of other pathways affected by ACVR1 R206H neofunction may be more important in immune cell types than previously thought. Finally, our finding that phosphorylated TAK1 normalized to total TAK1 was increased in both control and FOP monocytes LPS stimulation, with a trend toward an increase in FOP monocytes, hints that chronic stimulation of the TAK1 pathway and subsequent activation in the inflammasome (101) may be an important contributor to disease pathogenesis. Further experiments need to confirm the specific involvement of TAK1 in FOP cells. 
Although we were unable to fully characterize the increased subpopulation of $\mathrm{CD}_{14}{ }^{+} / \mathrm{CD} 16^{+}$intermediate cells in FOP subjects due to limited sample size and availability, these macrophages are known to be highly proinflammatory. Both increased and decreased cell frequency have been reported in several inflammatory conditions, such as sepsis, rheumatoid arthritis (53), or stroke $(102,103)$. Another cohort of FOP patients using a different FACS gating strategy did not find significant differences by monocytes subtype fractionation. However, FOP monocytes expressed higher levels of DNAX accessory molecule 1 (DNAM1), a protein important for monocyte migration through the endothelial barrier, again suggesting a role of monocytes in the early phases of FOP flare-ups (104). Further investigation of this population of $\mathrm{CD} 14^{+} \mathrm{CD} 16^{+}$proinflammatory macrophages is warranted.

Our findings have clinical implications for both FOP and nongenetic heterotopic ossification (Figure 9). First, increased immune activity may be present at baseline in FOP patients, reflecting a primed immune system sensitive to trauma. These cytokine and cellular profiles indicate that it may be possible to identify a biomarker risk panel for FOP bone formation and, potentially, other types of heterotopic ossification since FOP likely recapitulates other forms of heterotopic ossification. Although a recent study on 15 FOP patients suggested that there were little or no changes in serum cytokine markers (105), there are several key differences between our studies, including our collection of specimens that allowed for rapid processing and isolation of live cells for analysis, the use of a different and arguably more sensitive multiplex assays, and the inclusion of cell functional analyses to verify immune dysfunction.

Second, our findings suggest that the activated ACVR1 signaling has a critical effect on macrophage function and signaling activity. Some FOP patients seem to continue to acquire heterotopic bone, even in the absence of clinically identifiable flare events, or develop extensions of their heterotopic bone after the main flare event has clinically ended (14). Our findings suggest that a proinflammatory state may be present continuously, and they indicate that long-term/chronic therapy with immunosuppressive medication may be beneficial for managing FOP (33) or as an adjunct to steroid treatment (20). Indeed, a FOP patient treated by BM transplant for aplastic anemia had significant amelioration of his FOP symptoms until immunosuppression was discontinued (94). Immunomodulators such as imatinib may also have clinical benefits (106). However, whether this chronic bone formation can be targeted by specific regimens has yet to be determined.

Third, our findings that cytokine and monocyte/macrophage dysfunction were suppressed in flare-up patients who received standard-of-care treatments with steroids (approximately $2 \mathrm{mg} / \mathrm{kg}$ / day of prednisone; ref. 20) suggest that acute antiinflammatory treatments can modify the cytokine response. Our data likely reflect the clinical heterogeneity of FOP patients, where some FOP patients fail to respond to standard-ofcare high-dose steroid treatment and have continued inflammation and subsequent bone formation. Likewise, the cytokine profile heterogeneity in FOP patients at baseline without clinical flare symptoms may explain why some FOP patients show variability in sensitivity to trauma (14). Unfortunately, our study was unable to determine if the observed cytokine normalization is driven only by steroids, since few subjects did not receive standard-of-care treatment. Future longitudinal studies will help elucidate these mechanisms.

Our findings have critical implications for nongenetic forms of heterotopic ossification. Among the elevated cytokines found in FOP subjects' sera, or in LPS-stimulated FOP monocytes and/or macrophages, IL-3 was previously linked to heterotopic ossification in combat wounds (18), and IL-10 and MIP1- $\alpha$ were associated with heterotopic ossification due to high-energy trauma (19). VEGF, described to be increased in saliva 1 week after injury in a trauma-induced heterotopic ossification with hind limb Achilles' tenotomy and dorsal burn mouse model (26), was significantly increased in FOP monocytes and unstimulated M2 macrophage. Although IP10 (CXCL10) levels were significantly decrease in all FOP subjects, $C X C L 10$ gene expression was increased in a dose-response manner in LPS-stimulated FOP monocytes. Interestingly, IP10 wound effluent was previously shown to be associated and suggestive of heterotopic ossification development (19). Serum from FOP patients can enhance the osteoblast differentiation and BMP2 expression of mouse osteoblastic cells (107), further confirming the potential for systemic effects of the inflammatory system. Finally, a SNP in the TLR4 receptor has been associated with a decrease of trauma-induced heterotopic ossification formation (108). Thus, our results suggest that this cytokine network, and the changes we see in FOP, may have relevance to nongenetic causes of heterotopic ossification. Further work to elucidate the broader cytokine changes that occur with trauma will provide interesting comparisons with our results.

Our study has several important limitations. First, the need to collect FOP samples that could meet the processing and analytical requirements meant that we could only study a small cohort of FOP subjects who 
could come to our clinical site. Likewise, the small sample quantities and need for sequential analyses limited our ability to perform parallel studies where we could examine cytokines and cellular function together. In addition, our study opted to collect family controls to help decrease the influence of genetic background, which is a significant contributor to immune variability $(109,110)$. Although the control group was generally older than the FOP group, we still detected that standard-of-care steroid treatment in flaring FOP patients largely suppressed the cytokine changes, suggesting that antiinflammatory therapies may remain helpful for long-term treatment. We also were unable to perform longitudinal analyses on these samples; therefore, persistence of the cytokine changes over time remains unknown. Finally, the standard-of-care treatment regimen with steroids, the concomitant use of other medications, and the absence of longitudinal data to correlate cytokine profiles with long-term heterotopic bone formation currently limits our ability to directly link cytokine abnormalities to FOP-related bone formation. This is mitigated by the cellular changes that we found, but additional studies using model systems and in other forms of heterotopic ossification will help solidify our findings.

Together, our results demonstrate a proinflammatory state in FOP patients and a hyperresponsiveness of their peripheral blood monocytes and derived macrophages to inflammatory triggers. The remarkable overlap in cytokines/chemokines in FOP subjects and other forms of heterotopic ossification suggests that a common pathway may exist that could be potentially targeted as a generalized treatment for heterotopic ossification.

\section{Methods}

Subjects. Blood was collected from subjects undergoing blood collection for other purposes to reduce the chances of trauma-induced heterotopic ossification flares in FOP patients; thus, only small volumes of blood were available for research. Venous blood samples were carefully collected by experienced phlebotomistas from FOP subjects with no clinically active flare, FOP subjects with a clinically active flare, or control subjects who were relatives of the FOP patients (Figure 1). The limited sample volume meant that parallel studies of serum and cellular activities were usually not possible. Thus, details for each clinical sample for each study are described in Supplemental Tables 1-11. FOP patients from the No Flare group were not taking steroid treatments. All FOP patients taking steroids followed standard-of-care dosing (20). Nonsteroid medication treatments were also recorded; we did not identify any obvious link between those medications and changes in the measured parameters. Serum was isolated by centrifugation at 1,800 $\mathrm{g}$ for 30 minutes and sent to Eve Technologies for multiplex cytokine assessment. The concentration of Activin A and TGF- $\beta$ was quantified by Quantikine Elisa from R\&D Systems.

Primary monocyte isolation. Peripheral blood samples were collected by venipuncture in Vacuette K2E EDTA K2 tubes (catalog 1454428) and in BD Vacutainer CPT Cell preparation tubes (BD Biosciences, catalog 362761) with sodium citrate. For whole blood analysis, RBCs were lysed using RBC lysis buffer for human cells (Alfa Aesar, catalog J62990) twice for 20 minutes each. Remaining leukocytes were resuspended in FACS buffer (PBS/2\% FBS/2 mM EDTA) and analyzed by flow cytometry on a FACSAriaIII (BD Biosciences). For primary monocyte isolations, CPTs were centrifuged according to the manufacturer protocol. Buffy coat cells underwent magnetic sorting (MACS) using anti-CD14-coated beads (Miltenyi Biotec, catalog 130-059-201). Cells were analyzed by flow cytometry or cultured in monocyte media (RPMI1640 media supplemented with 10\% FBS and 1 mM glutamine; Gibco, Thermo Fisher Scientific). All antibodies used for FACs are listed in Supplemental Table 12.

Monocyte stimulation. After MACS purification for $\mathrm{CD}_{14}{ }^{+}$cells, primary monocytes were seeded at 10,000 cells per well in a 48-well plate. The next day, monocytes were stimulated with LPS $(10 \mathrm{ng} / \mathrm{ml}$, MilliporeSigma, catalog L2630), P3C (100 ng/ml, EMC Microcollections, catalog L200), FSL1 (100 ng/ ml, EMC Microcollections, catalog L7000), human Activin A (10 ng/ml, Peprotech, catalog 120-14E), or human BMP4 (10 ng/ml, Peprotech, catalog 120-05ET) for 24 hours. Supernatants were collected and RNA was extracted.

Monocyte differentiation into macrophages. $\mathrm{CD}^{+} 4^{+}$cells isolated by MACs were seeded at 10,000 cells per well in a 48-well plate or 300,000 cells per well in a 6-well plate and stimulated with GM-CSF ( $5 \mathrm{ng} / \mathrm{ml}$, Peprotech, catalog 300-03) or M-CSF (50 ng/ml, Peprotech, catalog 300-25) for 7 days. Additional media $(0.1$ or $1 \mathrm{ml})$ was added on day 3 to feed the cells. On day 7 , cells were stimulated with LPS $(10 \mathrm{ng} / \mathrm{ml}$, MilliporeSigma, catalog L2630). Supernatants were collected, and RNA was extracted.

Flow cytometry. Cells from whole blood were stained with anti-human CD16-AF488 (eBioscience, catalog 11-0168-42, clone eBioCB16), CD14-PE-Cy7 (eBioscience, catalog 25-0149-42, clone 61D3), HLA-DR-APC 
(eBioscience, catalog 17-9956-42, clone LN3), CD56-PE-Cy5 (eBioscience, catalog 35-0567-41, clone CMSSB), CD19-PE-Cy5(eBioscience, catalog 15-0199-42, clone HIB19), CD3-PE-Cy5 (Biolegend, catalog 300310, clone HIT3a), and CD66b-HRP (Biolegend, catalog B215969, clone G10F5) (Supplemental Table 12). Fluorescence intensity was determined for 100,000 cells total; percentages shown in figures are percent of living cells that fall within the gate shown. Monocyte subtypes were sorted with a FACSAriaIII (BD Biosciences). MACS purified CD14 ${ }^{+}$cells were stained for CD14-PE-Cy7, CD11b-AF488 (eBioscience, catalog 11-0118-42, clone ICRF44), CD206-APC (eBioscience, catalog 17-2069-42, clone 19.2), and CD163-PE (eBioscience, catalog 12-1639-42, clone eBioGHI/61) (Supplemental Table 12). Sytox blue (Thermo Fisher Scientific, catalog S34857) was used as a viability marker.

Real-time PCR and quantitative expression analysis. Gene expression of cytokines was used when only small cell populations were available. Total RNA was prepared using TRI Reagent (MilliporeSigma, catalog T9424) and processed with Arcturus PicoPure RNA isolation kit (Applied Biosystems, catalog KIT0204) as previously described (111). RNA $(0.2-0.5 \mu \mathrm{g})$ was reverse transcribed into cDNA with the VeriScript cDNA synthesis kit (Affymetrix, catalog 78070). cDNA was preamplified with GE PreAmp Master Mix (Fluidigm Inc., catalog 100-5580). Quantitative PCR (qPCR) was performed in technical triplicates with VeriQuest Probe qPCR Master Mix (Affymetrix, catalog 75650) on a Viia7 thermocycler (Invitrogen) or on a BioMark 48.48 dynamic array nanofluidic chip (Fluidigm Inc., catalog BMK-M-48.48) according to manufacturers' instructions. Taqman primers are listed in Supplemental Table 13. $\beta$-Actin was used for normalization as an endogenous control (112).

Immunofluorescence. Primary blood monocytes were seeded at 10,000 cells per well in an 8-chamber slide (Falcon), cultured in monocyte media for 3 days, and stimulated with LPS (10 ng/ml) for 24, 3, 1.5, 1 , and 0.5 hours; with Activin A $(50 \mathrm{ng} / \mathrm{ml})$ for 3 hours; or with BMP4 $(50 \mathrm{ng} / \mathrm{ml})$ for 3 hours. Cells were fixed for 15 minutes at room temperature in 4\% PFA (Thermo Fisher Scientific, catalog 28906 and were washed with PBS $3 \times$ for 5 minutes each time. The fixed cells were permeabilized and blocked at the same time with 5\% normal goat serum/0/3\% Triton X (MilliporeSigma, catalog T8787) in PBS for 1 hour at room temperature; they were then washed with PBS $3 \times$. Cells were incubated overnight at $4^{\circ} \mathrm{C}$ with primary antibodies: NF-кBp65 (Cell Signaling Technology, 1:400, catalog 3031), p38 (Cell Signaling Technology, 1:400, catalog 9211), phospho-SMAD 1/5 (Cell Signaling Technology, 1:400, catalog 9516), and rabbit phospho-P44/42 (Cell Signaling Technology, 1:400, catalog 4370, clone D13.14.4E). Cells were washed 3 times with PBS and incubated with secondary goat antimouse 568 (Invitrogen, 1:1,000) or goat anti-rabbit 568 (Invitrogen, 1:1,000, catalog A11034) for 30 minutes at room temperature in the dark. Cells were mounted with ProLong Gold Antifade Reagent with DAPI (Cell Signaling Technology, catalog 8961). Images were taken with a Keyence BZ-X700 microscope, and nuclear translocation was quantified with BZ-X analyzer software.

Phagocytosis assay. Primary monocytes (100,000 monocytes) were seeded onto a 12 -well plate and stimulated with GMCSF (5 ng/ml, Peprotech) or MCSF (50 ng/ml, Peprotech) for 7 days. Cells were starved for 1 hour. Bioparticles were opsonized and added to the cells according to manufacturer protocol (Thermo Fisher Scientific, Escherichia coli [K-12 strain], BioParticles, Alexa Fluor conjugate, catalog E13231). Wells were then imaged with a light microscope (Nikon Eclipse E800).

Western blot. Primary monocytes were stimulated for 30 minutes or 2 hours with LPS (10 ng/ $\mathrm{ml}$ ). Cells were harvested in Red Loading Buffer (Cell Signaling Technology, catalog 7723) supplemented with $1 \times$ protease and phosphatase inhibitor cocktail (Roche Diagnostics, catalogs 4906845001 and 4693132001). Primary monocyte-derived macrophages were serum starved for 1 hour prior to stimulation with LPS $(10 \mathrm{ng} / \mathrm{ml})$ or Activin A $(50 \mathrm{ng} / \mathrm{ml})$. Whole-cell lysates were prepared in Laemmli buffer (Bio-Rad, catalog 1610737) and resolved in 4-20\% tris-glycine gels (Bio-Rad, catalog 4561096). Primary antibodies for phospho-TAK1 (Cell Signaling Technology, catalog 9339), phospho-NF-кBp65 (Cell Signaling Technology, catalog 3031), phospho-p38 (Cell Signaling Technology, catalog 9211), phospho-SMAD1/5/9 (Cell Signaling Technology, catalog 13820, clone D5B10), and phospho-SMAD2 (Cell Signaling Technology, catalog 3108, clone 138D4) were used at a dilution of 1:1,000. Anti- $\beta$-actin antibody (Thermo Fisher Scientific, catalog MA5-15739, clone BA3R) was used at a dilution of $1: 10,000$. Binding was visualized with horseradish peroxidase-conjugated antibodies (Cell Signaling Technology, catalog 7074) and ECL substrate (Thermo Fisher Scientific, catalog 34095). An ImageQuant LAS 4000 (GE Healthcare) was used to image the blots, and quantifications were done using ImageJ (NIH) software. 
Statistics. All studies were performed with biological replicates as described in Supplemental Tables 1-11. The data were analyzed with GraphPad Prism v.7 software using 2-tailed Student's $t$ test and 2-way ANOVA Sidak or Tukey's multiple comparison tests. The Sidak test was used when comparing means between WT and FOP, and the Tukey test was used when means of both WT and FOP were compared together with other groups. The software $\mathrm{R}$ was used for heatmaps and PCA. $P<0.05$ were considered statistically significant.

Study approvals. All of the human study and sample collection procedures were reviewed and approved by the UCSF Committee on Human Research. All subjects provided informed consent prior to their participation in the study.

\section{Author contributions}

EB contributed design, collection, performed experiments, data assembly and analysis, interpretation, manuscript writing. BMM contributed data collection and performed experiments. CJC assisted with the flow cytometry strategy for the monocyte subtypes analysis and sorting. ANT performed mRNA extraction and cDNA synthesis. KLW saw FOP patients and collected clinical data. TVC contributed phlebotomy, blood collection, consenting forms, and clinical data assembly. TAM created heat maps, PCA analysis, and large data analysis. $\mathrm{MCH}$ and THMO provided monocyte isolation protocols and expertise, discussion on monocyte/macrophages markers. JH provided reagents and expertise and discussion on the TLRs. $\mathrm{MCN}$ assisted with the development and interpretation of data. ECH contributed conception and design of experiments; saw FOP patients; and contributed clinical data collection, financial support, data analysis and interpretation, and manuscript writing.

\section{Acknowledgments}

The authors would like to thank Wei Au, Koen Schepers, and Koen Braat for valuable technical comments and discussion. We thank Katherine Bigay, Jennifer Ho, and Shweta Hosakoppal for their technical assistance. This work was supported by the National Institute of Arthritis, Musculoskeletal, and Skin Disorders grant (R01 AR066735 to ECH), AR070155 (to MCN); the UCSF PREMIER program supported by P30AR070155, and the UCSF Core Center for Musculoskeletal Biology and Medicine (CCMBM) program supported by P30AR066262; a Doris Duke Charitable Foundation Clinical Scientist Development Award (2014099 to ECH); a Research Award from the March of Dimes (1-FY14-211 to ECH), the UCSF Department of Medicine and UCSF Cohort Development Grant (to ECH); the Radiant Hope Foundation (to ECH); the California Institute for Regenerative Medicine Fellowship Program to UCSF (TG201153 to EB); the National Institutes of Health (T32DK007161 to CJC and F32HD091025 to KLW), an Alpha-Omega-Alpha Carolyn L. Kuckein Summer Research Fellowship (to AT), a Howard Hughes Medical Institute Medical Research Fellowship (to AT), the Russell/Engleman Arthritis Center at UCSF (to $\mathrm{MCN}$ ), and the Department of Veteran's Affairs Health Care System (to MCN).

Address correspondence to: Emilie Barruet or Edward Hsiao, Division of Endocrinology and Metabolism, Department of Medicine, Institute for Human Genetics, University of California at San Francisco, 513 Parnassus Avenue, HSE 901G, San Francisco, California 94143-0794, USA. Phone: 415.476.9732; Email: Emilie.Barruet@ucsf.edu (EB); Edward.Hsiao@ucsf.edu (EH).

\footnotetext{
1. Eming SA, Wynn TA, Martin P. Inflammation and metabolism in tissue repair and regeneration. Science. 2017;356(6342):1026-1030.

2. Frangogiannis NG. The Inflammatory Response in Tissue Repair. In: Cavaillon JM, Singer M, eds. Inflammation: From Molecular and Cellular Mechanisms to the Clinic. Hoboken, NJ: Wiley; 2017:1517-1537.

3. Mountziaris PM, Mikos AG. Modulation of the inflammatory response for enhanced bone tissue regeneration. Tissue Eng Part B Rev. 2008;14(2):179-186.

4. Charles JF, Nakamura MC. Bone and the innate immune system. Curr Osteoporos Rep. 2014;12(1):1-8.

5. Claes L, Recknagel S, Ignatius A. Fracture healing under healthy and inflammatory conditions. Nat Rev Rheumatol. 2012;8(3):133-143.

6. Champagne CM, Takebe J, Offenbacher S, Cooper LF. Macrophage cell lines produce osteoinductive signals that include bone morphogenetic protein-2. Bone. 2002;30(1):26-31.

7. Convente MR, Wang H, Pignolo RJ, Kaplan FS, Shore EM. The immunological contribution to heterotopic ossification disorders. Curr Osteoporos Rep. 2015;13(2):116-124.

8. McCarthy EF, Sundaram M. Heterotopic ossification: a review. Skeletal Radiol. 2005;34(10):609-619.

9. Kocic M, Lazovic M, Mitkovic M, Djokic B. Clinical significance of the heterotopic ossification after total hip arthroplasty.
} 
Orthopedics. 2010;33(1):16.

10. Leung C, et al. Clinical significance of heterotopic ossification in cervical disc replacement: a prospective multicenter clinical trial. Neurosurgery. 2005;57(4):759-763

11. Hurvitz EA, Mandac BR, Davidoff G, Johnson JH, Nelson VS. Risk factors for heterotopic ossification in children and adolescents with severe traumatic brain injury. Arch Phys Med Rehabil. 1992;73(5):459-462.

12. Potter BK, Burns TC, Lacap AP, Granville RR, Gajewski DA. Heterotopic ossification following traumatic and combat-related amputations. Prevalence, risk factors, and preliminary results of excision. J Bone Joint Surg Am. 2007;89(3):476-486.

13. Sullivan MP, Torres SJ, Mehta S, Ahn J. Heterotopic ossification after central nervous system trauma: A current review. Bone Joint Res. 2013;2(3):51-57.

14. Pignolo RJ, et al. The Natural History of Flare-Ups in Fibrodysplasia Ossificans Progressiva (FOP): A Comprehensive Global Assessment. J Bone Miner Res. 2016;31(3):650-656.

15. Yao Y, et al. Inhibition of bone morphogenetic proteins protects against atherosclerosis and vascular calcification. Circ Res. 2010;107(4):485-494.

16. Chen W, Ten Dijke P. Immunoregulation by members of the TGFß superfamily. Nat Rev Immunol. 2016;16(12):723-740.

17. Chen W, Wahl SM. TGF-beta: receptors, signaling pathways and autoimmunity. Curr Dir Autoimmun. 2002;5:62-91.

18. Forsberg JA, Potter BK, Polfer EM, Safford SD, Elster EA. Do inflammatory markers portend heterotopic ossification and wound failure in combat wounds? Clin Orthop Relat Res. 2014;472(9):2845-2854.

19. Evans $\mathrm{KN}$, et al. Inflammatory cytokine and chemokine expression is associated with heterotopic ossification in high-energy penetrating war injuries. J Orthop Trauma. 2012;26(11):e204-e213.

20. Kaplan FS, Shore EM, Pignolo RJ (eds), Hsiao EC, The International Clinical Consortium on FOP. The medical management of fibrodysplasia ossificans progressiva: current treatment considerations. Clin Proc Intl Clin Consort FOP. 2011;4:1-100

21. Kan SL, et al. Nonsteroidal Anti-inflammatory Drugs as Prophylaxis for Heterotopic Ossification after Total Hip Arthroplasty: A Systematic Review and Meta-Analysis. Medicine (Baltimore). 2015;94(18):e828.

22. Beckmann JT, Wylie JD, Potter MQ, Maak TG, Greene TH, Aoki SK. Effect of Naproxen Prophylaxis on Heterotopic Ossification Following Hip Arthroscopy: A Double-Blind Randomized Placebo-Controlled Trial. J Bone Joint Surg Am. 2015;97(24):2032-2037.

23. Rath E, et al. Selective COX-2 Inhibitors Significantly Reduce the Occurrence of Heterotopic Ossification After Hip Arthroscopic Surgery. Am J Sports Med. 2016;44(3):677-681.

24. Convente MR, et al. Depletion of Mast Cells and Macrophages Impairs Heterotopic Ossification in an Acvr1R206H Mouse Model of Fibrodysplasia Ossificans Progressiva. J Bone Miner Res. 2018;33(2):269-282.

25. Wang X, et al. Inhibition of overactive TGF- $\beta$ attenuates progression of heterotopic ossification in mice. Nat Commun. 2018;9(1):551.

26. Sung Hsieh HH, et al. Evaluation of Salivary Cytokines for Diagnosis of both Trauma-Induced and Genetic Heterotopic Ossification. Front Endocrinol (Lausanne). 2017;8:74.

27. Raggatt LJ, et al. Fracture healing via periosteal callus formation requires macrophages for both initiation and progression of early endochondral ossification. Am J Pathol. 2014;184(12):3192-3204.

28. Song GA, et al. Molecular consequences of the ACVR1(R206H) mutation of fibrodysplasia ossificans progressiva. J Biol Chem. 2010;285(29):22542-22553.

29. Shore EM, et al. A recurrent mutation in the BMP type I receptor ACVR1 causes inherited and sporadic fibrodysplasia ossificans progressiva. Nat Genet. 2006;38(5):525-527.

30. Hatsell SJ, et al. ACVR1R206H receptor mutation causes fibrodysplasia ossificans progressiva by imparting responsiveness to activin A. Sci Transl Med. 2015;7(303):303ra137.

31. Hino K, et al. Neofunction of ACVR1 in fibrodysplasia ossificans progressiva. Proc Natl Acad Sci USA. 2015;112(50):15438-15443.

32. Barruet E, et al. The ACVR1 R206H mutation found in fibrodysplasia ossificans progressiva increases human induced pluripotent stem cell-derived endothelial cell formation and collagen production through BMP-mediated SMAD1/5/8 signaling. Stem Cell Res Ther. 2016;7(1):115.

33. Pignolo RJ, Shore EM, Kaplan FS. Fibrodysplasia ossificans progressiva: clinical and genetic aspects. Orphanet J Rare Dis. 2011;6:80.

34. Chakkalakal SA, et al. An Acvr1 R206H knock-in mouse has fibrodysplasia ossificans progressiva. J Bone Miner Res. 2012;27(8):1746-1756.

35. Shore EM, Feldman GJ, Xu M, Kaplan FS. The genetics of fibrodysplasia ossificans progressiva. Clin Rev Bone Miner Metab. 2005;3(3):201-204.

36. Baujat G, et al. Prevalence of fibrodysplasia ossificans progressiva (FOP) in France: an estimate based on a record linkage of two national databases. Orphanet J Rare Dis. 2017;12(1):123.

37. Metcalf D. Hematopoietic cytokines. Blood. 2008;111(2):485-491.

38. Chen Z, et al. The novel role of IL-7 ligation to IL-7 receptor in myeloid cells of rheumatoid arthritis and collagen-induced arthritis. J Immunol. 2013;190(10):5256-5266.

39. Arango Duque G, Descoteaux A. Macrophage cytokines: involvement in immunity and infectious diseases. Front Immunol. 2014;5:491.

40. Oude Nijhuis CS, Vellenga E, Daenen SM, Kamps WA, De Bont ES. Endothelial cells are main producers of interleukin 8 through Toll-like receptor 2 and 4 signaling during bacterial infection in leukopenic cancer patients. Clin Diagn Lab Immunol. 2003;10(4):558-563.

41. Yeh M, et al. Increased transcription of IL-8 in endothelial cells is differentially regulated by TNF-alpha and oxidized phospholipids. Arterioscler Thromb Vasc Biol. 2001;21(10):1585-1591.

42. Wicks IP, Roberts AW. Targeting GM-CSF in inflammatory diseases. Nat Rev Rheumatol. 2016;12(1):37-48.

43. Iyer SS, Cheng G. Role of interleukin 10 transcriptional regulation in inflammation and autoimmune disease. Crit Rev Immunol. 2012;32(1):23-63.

44. Wang J, et al. Injury-induced MRP8/MRP14 stimulates IP-10/CXCL10 in monocytes/macrophages. FASEB J. 2015;29(1):250-262. 
45. Ancuta P, et al. Fractalkine preferentially mediates arrest and migration of CD16+ monocytes. J Exp Med. 2003;197(12):1701-1707.

46. White GE, McNeill E, Channon KM, Greaves DR. Fractalkine promotes human monocyte survival via a reduction in oxidative stress. Arterioscler Thromb Vasc Biol. 2014;34(12):2554-2562.

47. Mionnet $\mathrm{C}$, et al. CX3CR1 is required for airway inflammation by promoting $\mathrm{T}$ helper cell survival and maintenance in inflamed lung. Nat Med. 2010;16(11):1305-1312.

48. Taylor PC, Feldmann M. Anti-TNF biologic agents: still the therapy of choice for rheumatoid arthritis. Nat Rev Rheumatol. 2009;5(10):578-582.

49. Jones BA, Beamer M, Ahmed S. Fractalkine/CX3CL1: a potential new target for inflammatory diseases. Mol Interv. 2010;10(5):263-270.

50. Goswami R, Kaplan MH. A brief history of IL-9. J Immunol. 2011;186(6):3283-3288.

51. Abeles RD, et al. CD14, CD16 and HLA-DR reliably identifies human monocytes and their subsets in the context of pathologically reduced HLA-DR expression by CD14(hi) /CD16(neg) monocytes: Expansion of CD14(hi) /CD16(pos) and contraction of CD14(lo) /CD16(pos) monocytes in acute liver failure. Cytometry A. 2012;81(10):823-834.

52. Mukherjee R, Kanti Barman P, Kumar Thatoi P, Tripathy R, Kumar Das B, Ravindran B. Non-Classical monocytes display inflammatory features: Validation in Sepsis and Systemic Lupus Erythematous. Sci Rep. 2015;5:13886.

53. Yang J, Zhang L, Yu C, Yang XF, Wang H. Monocyte and macrophage differentiation: circulation inflammatory monocyte as biomarker for inflammatory diseases. Biomark Res. 2014;2(1):1.

54. Rinehart JJ, Sagone AL, Balcerzak SP, Ackerman GA, LoBuglio AF. Effects of corticosteroid therapy on human monocyte function. N Engl J Med. 1975;292(5):236-241.

55. Torossian F, et al. Macrophage-derived oncostatin M contributes to human and mouse neurogenic heterotopic ossifications. JCI Insight. 2017;2(21):e96034.

56. Wang H, Behrens EM, Pignolo RJ, Kaplan FS. ECSIT links TLR and BMP signaling in FOP connective tissue progenitor cells. Bone. 2018;109:201-209

57. Shaulian E, Karin M. AP-1 as a regulator of cell life and death. Nat Cell Biol. 2002;4(5):E131-E136.

58. Chong SZ, et al. CXCR4 identifies transitional bone marrow premonocytes that replenish the mature monocyte pool for peripheral responses. J Exp Med. 2016;213(11):2293-2314.

59. Park BS, Lee JO. Recognition of lipopolysaccharide pattern by TLR4 complexes. Exp Mol Med. 2013;45:e66.

60. Jaguin M, Houlbert N, Fardel O, Lecureur V. Polarization profiles of human M-CSF-generated macrophages and comparison of M1-markers in classically activated macrophages from GM-CSF and M-CSF origin. Cell Immunol. 2013;281(1):51-61.

61. Valdimarsdottir G, et al. Stimulation of Id1 expression by bone morphogenetic protein is sufficient and necessary for bone morphogenetic protein-induced activation of endothelial cells. Circulation. 2002;106(17):2263-2270.

62. Zhou D, et al. Macrophage polarization and function with emphasis on the evolving roles of coordinated regulation of cellular signaling pathways. Cell Signal. 2014;26(2):192-197.

63. Lamb DJ, Modjtahedi H, Plant NJ, Ferns GA. EGF mediates monocyte chemotaxis and macrophage proliferation and EGF receptor is expressed in atherosclerotic plaques. Atherosclerosis. 2004;176(1):21-26.

64. Heil M, et al. Vascular endothelial growth factor (VEGF) stimulates monocyte migration through endothelial monolayers via increased integrin expression. Eur J Cell Biol. 2000;79(11):850-857.

65. Avraham-Davidi I, et al. On-site education of VEGF-recruited monocytes improves their performance as angiogenic and arteriogenic accessory cells. J Exp Med. 2013;210(12):2611-2625.

66. Eubank TD, Galloway M, Montague CM, Waldman WJ, Marsh CB. M-CSF induces vascular endothelial growth factor production and angiogenic activity from human monocytes. J Immunol. 2003;171(5):2637-2643.

67. Itaya H, Imaizumi T, Yoshida H, Koyama M, Suzuki S, Satoh K. Expression of vascular endothelial growth factor in human monocyte/macrophages stimulated with lipopolysaccharide. Thromb Haemost. 2001;85(1):171-176.

68. Irie T, Muta T, Takeshige K. TAK1 mediates an activation signal from toll-like receptor(s) to nuclear factor-kappaB in lipopolysaccharide-stimulated macrophages. FEBS Lett. 2000;467(2-3):160-164.

69. Shim JH, et al. TAK1 is an essential regulator of BMP signalling in cartilage. EMBO J. 2009;28(14):2028-2041.

70. Wynn TA, Barron L. Macrophages: master regulators of inflammation and fibrosis. Semin Liver Dis. 2010;30(3):245-257.

71. Tidball JG, Wehling-Henricks M. Shifts in macrophage cytokine production drive muscle fibrosis. Nat Med. 2015;21(7):665-666.

72. Mundy GR. Osteoporosis and inflammation. Nutr Rev. 2007;65(12 Pt 2):S147-S151.

73. Redlich K, Smolen JS. Inflammatory bone loss: pathogenesis and therapeutic intervention. Nat Rev Drug Discov. 2012;11(3):234-250.

74. Lounev VY, et al. Identification of progenitor cells that contribute to heterotopic skeletogenesis. J Bone Joint Surg Am. 2009;91(3):652-663

75. Medici D, Shore EM, Lounev VY, Kaplan FS, Kalluri R, Olsen BR. Conversion of vascular endothelial cells into multipotent stem-like cells. Nat Med. 2010;16(12):1400-1406.

76. Dey D, et al. Two tissue-resident progenitor lineages drive distinct phenotypes of heterotopic ossification. Sci Transl Med. 2016;8(366):366ra163.

77. Wosczyna MN, Biswas AA, Cogswell CA, Goldhamer DJ. Multipotent progenitors resident in the skeletal muscle interstitium exhibit robust BMP-dependent osteogenic activity and mediate heterotopic ossification. J Bone Miner Res. 2012;27(5):1004-1017.

78. Lees-Shepard JB, et al. Activin-dependent signaling in fibro/adipogenic progenitors causes fibrodysplasia ossificans progressiva. Nat Commun. 2018;9(1):471.

79. Tete S, et al. Interleukin-9 and mast cells. J Biol Regul Homeost Agents. 2012;26(3):319-326.

80. Halova I, Draberova L, Draber P. Mast cell chemotaxis - chemoattractants and signaling pathways. Front Immunol. 2012;3:119.

81. Kitaura M, et al. Molecular cloning of human eotaxin, an eosinophil-selective CC chemokine, and identification of a specific eosinophil eotaxin receptor, CC chemokine receptor 3. J Biol Chem. 1996;271(13):7725-7730.

82. Ye J, Kohli LL, Stone MJ. Characterization of binding between the chemokine eotaxin and peptides derived from the chemokine receptor CCR3. J Biol Chem. 2000;275(35):27250-27257.

83. Sawant KV, et al. Chemokine CXCL1 mediated neutrophil recruitment: Role of glycosaminoglycan interactions. Sci Rep. 
2016;6:33123

84. De Filippo K, et al. Mast cell and macrophage chemokines CXCL1/CXCL2 control the early stage of neutrophil recruitment during tissue inflammation. Blood. 2013;121(24):4930-4937.

85. Arend WP, Malyak M, Guthridge CJ, Gabay C. Interleukin-1 receptor antagonist: role in biology. Annu Rev Immunol. 1998;16:27-55.

86. Miossec P, Kolls JK. Targeting IL-17 and TH17 cells in chronic inflammation. Nat Rev Drug Discov. 2012;11(10):763-776.

87. Wynn TA, Chawla A, Pollard JW. Macrophage biology in development, homeostasis and disease. Nature. 2013;496(7446):445455.

88. Jabri B, Abadie V. IL-15 functions as a danger signal to regulate tissue-resident T cells and tissue destruction. Nat Rev Immunol. 2015;15(12):771-783.

89. Kaplan FS, Pignolo RJ, Shore EM. The FOP metamorphogene encodes a novel type I receptor that dysregulates BMP signaling. Cytokine Growth Factor Rev. 2009;20(5-6):399-407.

90. Edderkaoui B. Potential Role of Chemokines in Fracture Repair. Front Endocrinol (Lausanne). 2017;8:39

91. Martinet Y, Bitterman PB, Mornex JF, Grotendorst GR, Martin GR, Crystal RG. Activated human monocytes express the c-sis proto-oncogene and release a mediator showing PDGF-like activity. Nature. 1986;319(6049):158-160.

92. Kusumbe AP, Ramasamy SK, Adams RH. Coupling of angiogenesis and osteogenesis by a specific vessel subtype in bone. Nature. 2014;507(7492):323-328.

93. Seok J, et al. Genomic responses in mouse models poorly mimic human inflammatory diseases. Proc Natl Acad Sci USA. 2013;110(9):3507-3512.

94. Kaplan FS, et al. Hematopoietic stem-cell contribution to ectopic skeletogenesis. J Bone Joint Surg Am. 2007;89(2):347-357.

95. Yu L, Wang L, Chen S. Endogenous toll-like receptor ligands and their biological significance. J Cell Mol Med. 2010;14(11):2592-2603.

96. Ahn J, Feldman G, Terry L, Shore EM, Kaplan FS. Exoneration of NF-kappaB dysregulation in fibrodysplasia ossificans progressiva. Clin Orthop Relat Res. 2003;(406):205-213.

97. Zhang Q, Lenardo MJ, Baltimore D. 30 Years of NF-кB: A Blossoming of Relevance to Human Pathobiology. Cell. 2017;168(12):37-57.

98. Lawrence T. The nuclear factor NF-kappaB pathway in inflammation. Cold Spring Harb Perspect Biol. 2009;1(6):a001651.

99. Augeri DJ, Langenfeld E, Castle M, Gilleran JA, Langenfeld J. Inhibition of BMP and of TGF $\beta$ receptors downregulates expression of XIAP and TAK1 leading to lung cancer cell death. Mol Cancer. 2016;15:27.

100. Gunnell LM, et al. TAK1 regulates cartilage and joint development via the MAPK and BMP signaling pathways. $J$ Bone Miner Res. 2010;25(8):1784-1797.

101. Malireddi RKS, et al. TAK1 restricts spontaneous NLRP3 activation and cell death to control myeloid proliferation. J Exp Med. 2018;215(4):1023-1034.

102. Poehlmann H, Schefold JC, Zuckermann-Becker H, Volk HD, Meisel C. Phenotype changes and impaired function of dendritic cell subsets in patients with sepsis: a prospective observational analysis. Crit Care. 2009;13(4):R119.

103. Urra X, et al. Monocyte subtypes predict clinical course and prognosis in human stroke. J Cereb Blood Flow Metab. 2009;29(5):994-1002.

104. Del Zotto G, et al. Peripheral Blood Mononuclear Cell Immunophenotyping in Fibrodysplasia Ossificans Progressiva Patients Evidence for Monocyte DNAM1 Up-regulation. Cytometry B Clin Cytom. 2018;94(4):613-622.

105. Hildebrand L, et al. Trace element and cytokine concentrations in patients with Fibrodysplasia Ossificans Progressiva (FOP): A case control study. J Trace Elem Med Biol. 2017;39:186-192.

106. Kaplan FS, et al. Early clinical observations on the use of imatinib mesylate in FOP: A report of seven cases. Bone. 2018;109:276-280.

107. Hisa I, Kawara A, Katagiri T, Sugimoto T, Kaji H. Effects of Serum from a Fibrodysplasia Ossificans Progressiva Patient on Osteoblastic Cells. Open J Endocr Metab Dis. 2012;2(1):6.

108. Mitchell EJ, Canter J, Norris P, Jenkins J, Morris J. The genetics of heterotopic ossification: insight into the bone remodeling pathway. J Orthop Trauma. 2010;24(9):530-533.

109. Nédélec Y, et al. Genetic Ancestry and Natural Selection Drive Population Differences in Immune Responses to Pathogens. Cell. 2016;167(3):657-669.e21.

110. Kim S, et al. Characterizing the genetic basis of innate immune response in TLR4-activated human monocytes. Nat Commun. 2014;5:5236.

111. Schepers K, Hsiao EC, Garg T, Scott MJ, Passegué E. Activated Gs signaling in osteoblastic cells alters the hematopoietic stem cell niche in mice. Blood. 2012;120(17):3425-3435.

112. Piehler AP, Grimholt RM, Ovstebø R, Berg JP. Gene expression results in lipopolysaccharide-stimulated monocytes depend significantly on the choice of reference genes. BMC Immunol. 2010;11:21. 